\title{
Classicality of the order parameter during a phase transition
}

\author{
Fernando C. Lombardo ${ }^{1,2} *$, Francisco D. Mazzitelli ${ }^{1 \dagger}$, and Diana Monteoliva ${ }^{1 \ddagger}$ \\ ${ }^{1}$ Departamento de Física, Facultad de Ciencias Exactas y Naturales \\ Universidad de Buenos Aires - Ciudad Universitaria, Pabellón I \\ 1428 Buenos Aires, Argentina \\ ${ }^{2}$ Blackett Laboratory, Imperial College, London SW7 2BZ
}

\begin{abstract}
We analyze the quantum to classical transition of the order parameter in second order phase transitions. We consider several toy models in non relativistic quantum mechanics. We study the dynamical evolution of a wave packet initially peaked around a local maximum of the potential using variational approximations and also exact numerical results. The influence of the environment on the evolution of the density matrix and the Wigner function is analyzed in great detail. We also discuss the relevance of our results to the analysis of phase transitions in field theory. In particular, we argue that previous results about classicality of the order parameter in $O(N)$ models may be consequences of the large $N$ approximation.
\end{abstract}

PACS numbers: 05.70.Fh, 03.70.+k, 05.40.Jc

*Electronic address: f.lombardo@ic.ac.uk

†Electronic address: fmazzi@df.uba.ar

‡Electronic address: monteoli@df.uba.ar 


\section{INTRODUCTION}

The emergence of classical behaviour from a quantum system is a problem of interest in many branches of physics [1]. As is well known, the quantum to classical transition involves two necessary and related conditions: correlations, i.e. the Wigner function of a quantum system should have a peak at the classical trajectories; and decoherence, that is, there should be no interference between classical trajectories. To study quantitatively the emergence of classicality, it is essential to consider the interaction of the system with its environment, since both the loss of quantum coherence and the onset of classical correlations depend strongly on this interaction [2]. Using this point of view, classicality is an emergent property of an open quantum system. The strength of the coupling between system and environment sets the decoherence time which, roughly speaking, indicates the timescale after which the system can be considered as classical.

Our concern in this paper will be the analysis of the quantum to classical transition of the order parameter during second order phase transitions, in which the effective potential has a local maximum. In condensed matter physics, there are several systems in which the dynamics of phase transitions can be studied experimentally (superfluids for example) [3]. In the standard big-bang cosmological model, phase transitions occur at the GUT and EW scales. During these phase transitions topological defects are inevitable, and they may have played a fundamental role in the formation of large scale structure (strings) [3]. Moreover, superabundance of some topological defects may contradict observational evidence (magnetic monopoles). In order to solve this and related problems it is widely accepted that, before the radiation dominated era, the Universe expanded exponentially (inflationary period). This exponential expansion takes place during a second order phase transition.

In all the above mentioned examples there is an order parameter which evolves from the false to the true vacuum of the theory: the Higgs fields in GUT and EW phase transitions, the inflaton field(s) in inflationary models, etc. Although these are quantum scalar fields with vanishing mean value (due to the symmetry of the initial quantum state), the order parameter is usually treated as a classical object. The classical behaviour is fundamental to define and count the topological defects [ []], and to justify the fact that some gauge and fermion fields aquire masses.

In the present work, we will analyze the classicality of the order parameter during a second order phase transition. Of course, in a realistic model one should address this problem in the context of quantum field theory. In fact, a possible approach would be to follow the analysis started by two of us in Ref. [5], where we studied the emergence of classical inhomogeneities from quantum fluctuations for a self-interacting quantum scalar field. We have investigated there the decoherence induced on the long-wavelength field modes by coarse graining the field modes with wavelength shorter than a critical value, in order to show how the system becomes classical due to the interaction with its environment (in that case composed by the short-wavelength field modes of the same field). The classicality of the order parameter could be analyzed along the same lines by considering a model with spontaneous symmetry breaking.

This is an extremely difficult problem because, as has been pointed out in the literature, and as we will stress in what follows, non perturbative and non Gaussian effects are relevant in the quantum to classical transition of the order parameter. In field theory, it is very 
difficult to go beyond perturbative or mean field methods (Hartree, $1 / N$, etc). For this reason, in the present paper we will be mainly concerned with toy models: closed and open systems [6] in non relativistic quantum mechanics. We will study the spread of a wave packet initially centered around the local maximum of a double well potential, paying particular attention to the influence of the environment on the Wigner function and on the reduced density matrix. We will also discuss the relevance of our results to the analysis of the field theory phase transitions.

The paper is organized as follows. In the next section we review the approach of Guth and $\mathrm{Pi}$ to describe the initial stages of the quantum dynamics of the phase transition using an inverted harmonic oscillator. We consider the upside down harmonic oscillator with and without environment, in order to emphazise the relevance of the environment in the quantum to classical transition. In section III we study the evolution of a wave packet initially centered on the top of a double well potential. We describe a Gaussian variational calculation and an improved version of it. We find that, in the Gaussian approximation, the Wigner function is positive for all times but it does not describe classical correlations unless the system is coupled to an environment. The Gaussian approach breaks down as the wave packet spreads out and explores the minimum of the potential. The improved variational approximation describes the dynamics of the system beyond that point. However, as the wave function is not Gaussian, the Wigner function is no longer positive. In section IV we describe the exact numerical evaluation of the evolution of the wave packet. We show that, as the coupling between the system and the environment increases, the decoherence time decreases. Due to the nonlinearities of the potential, when the coupling vanishes there is no classical limit, not even classical correlations. In section $\mathrm{V}$ we analyze previous works on the quantum to classical transition in field theory in the light of the results for the toy models.

\section{THE INVERTED HARMONIC OSCILLATOR: ROLE OF THE ENVIRONMENT}

In a cosmological scenario, at very high temperatures the effective potential for a scalar field $\phi$ has a minimum at $\phi=0$. As the temperature decreases this minimum becomes unstable, and the stable minima move to a nonvanishing value of the field. During the phase transition, the system evolves to its true vacuum.

A sudden quench phase transition can be described by a field theory in which there is an instantaneous change of sign in the mass term of the scalar field

$$
S[\phi]=\int d^{4} x\left[\frac{1}{2} \partial_{\mu} \phi \partial^{\mu} \phi-\frac{1}{2} m(t)^{2} \phi^{2}-\frac{1}{4 !} \lambda \phi^{4}\right],
$$

where $m^{2}(t)=\mu^{2}>0$ for $t<t_{0}$ and $m^{2}(t)=-\mu^{2}$ for $t>t_{0}$ (we will take $t_{0}=0$ for simplicity). This change of sign in $m^{2}(t)$ breaks the global symmetry for $t>0$.

Guth and Pi [7] considered an upside down harmonic oscillator as a toy model to describe the quantum behaviour of this unstable system

$$
S[x]=\int_{0}^{t} d s \frac{1}{2} M\left(\dot{x}^{2}+\Omega_{0}^{2} x^{2}\right) .
$$

This toy model should be a good approximation for the early time evolution of the phase transition, as long as one can neglect the non-linearities of the potential. 
If the initial wave function is Gaussian, it will remain Gaussian for all times (with time dependent parameters that set its amplitude and spread). The density matrix will be of the form

$$
\rho\left(x, x^{\prime}, t\right)=N(t) e^{-A(t) x^{2}-B(t) x^{\prime 2}},
$$

where $N(t)$ is a time-dependent real normalization function; $A(t)$ and $B(t)$ are time dependent complex coefficients, which satisfy $A=a+i b=B^{*}$. Using new variables $\Sigma=\left(x+x^{\prime}\right) / 2$ and $\Delta=\left(x-x^{\prime}\right) / 2$, the density matrix can be re-written as

$$
\rho(t, \Sigma, \Delta)=N(t) e^{-2 a \Delta^{2}} e^{-2 a \Sigma^{2}} e^{-4 i b \Sigma \Delta} .
$$

For such a density matrix, the asociated Wigner function is also Gaussian and can be exactly evaluated as (here and what follows we set $\hbar \equiv 1$ )

$$
W(x, p, t)=\frac{1}{2 \pi} \int_{-\infty}^{+\infty} d y e^{i p y} \rho\left(x+\frac{y}{2}, x-\frac{y}{2}\right)=\frac{1}{\pi} e^{-2 a x^{2}} e^{-\frac{(p-2 x b)^{2}}{2 a}} .
$$

The coefficient $2 a$ gives the spread of the Wigner distribution around the classical trajectory, and, at the same time, $(2 a)^{-1}$ measures the importance of the non-diagonal terms in the density matrix. As is well known, there is a compromise between the spread of the Wigner function and the diagonalization of the density matrix: as one becomes peaked the other becomes non diagonal [8].

In Fig. 1, we show the time dependence of $2 a$ obtained from the Schröedinger equation. It is an exponentially decreasing function. Therefore, although the density matrix is nondiagonal, the Wigner distribution becomes peaked around the classical trajectory for long times. The unstable quantum evolution shows classical behaviour, in the sense that one can obtain a classical probability distribution for the unstable particle [7]. Strictly speaking, we do not have classical limit but "classical correlations", because the density matrix is not diagonal. As has been recently pointed out by Kiefer et al [10], this may be enough for the quantum to classical transition of free field fluctuations in a cosmological setting. However, as we will see in the next sections, this is not true for the double well potential.

The classical limit exhibited by the quantum particle in the upside down potential requires the coefficient $2 a$ to reach its minimum value. This limit is obviously reached at large times, when the particle is far away from the potential top. The "correlation time" depends on the parameters of the potential. As we will now see, the presence of an environment changes drastically this situation.

Let us consider the unstable quantum particle (characterized by its mass $M$ and its bare frequency $\Omega_{0}$ ) linearly coupled to an environment composed by an infinite set of harmonic oscillators (of mass $m_{n}$ and frequency $\omega_{n}$ ). We may write the total action corresponding to the system-environment model as

$$
\begin{aligned}
S\left[x, q_{n}\right] & =S[x]+S\left[q_{n}\right]+S_{\mathrm{int}}\left[x, q_{n}\right] \\
& =\int_{0}^{t} d s\left[\frac{1}{2} M\left(\dot{x}^{2}+\Omega_{0}^{2} x^{2}\right)+\sum_{n} \frac{1}{2} m_{n}\left(\dot{q}_{n}^{2}-\omega_{n}^{2} q_{n}^{2}\right)\right]-\sum_{n} C_{n} x q_{n},
\end{aligned}
$$

where $x$ and $q_{n}$ are the coordinates of the particle and the oscillators, respectively. The unstable particle is coupled linearly to each oscillator with strength $C_{n}$. 
The relevant objects to analize the quantum to classical transition in this model are the reduced density matrix, and the associated Wigner function

$$
\begin{aligned}
& \rho_{\mathrm{r}}\left(x, x^{\prime}, t\right)=\int d q_{n} \rho\left(x, q_{n}, x^{\prime}, q_{n}, t\right) \\
& W_{\mathrm{r}}(x, p, t)=\frac{1}{2 \pi} \int_{-\infty}^{+\infty} d y e^{i p y} \rho_{\mathrm{r}}\left(x+\frac{y}{2}, x-\frac{y}{2}, t\right) .
\end{aligned}
$$

The reduced density matrix satisfies a master equation. Hu-Paz-Zhang [6] have evaluated the master equation for the quantum Brownian motion problem. Following the same procedure, we can write the master equation for the unstable particle [9]. As the coupling between system and environment is lineal, the result is exact, and can be easily obtained it replacing $\Omega_{0}$ by $i \Omega_{0}$ in the $\mathrm{Hu}-\mathrm{Paz}-\mathrm{Zhang}$ result

$$
\dot{\rho}_{\mathrm{r}}=-i\left[H_{\mathrm{syst}}-\frac{1}{2} M \tilde{\Omega}^{2}(t), \rho_{\mathrm{r}}\right]+2 i \gamma(t)\left[x,\left\{p, \rho_{\mathrm{r}}\right\}\right]-D(t)\left[x,\left[x, \rho_{\mathrm{r}}\right]\right]-f(t)\left[x,\left[p, \rho_{\mathrm{r}}\right]\right] .
$$

The time dependent coefficients are given by

$$
\begin{aligned}
\tilde{\Omega}^{2}(t) & =-\frac{2}{M} \int_{0}^{t} d t^{\prime} \cosh \left(\Omega_{0} t^{\prime}\right) \eta\left(t^{\prime}\right) \\
\gamma(t) & =-\frac{1}{2 M \Omega_{0}} \int_{0}^{t} d t^{\prime} \sinh \left(\Omega_{0} t^{\prime}\right) \eta\left(t^{\prime}\right) \\
D(t) & =\int_{0}^{t} d t^{\prime} \cosh \left(\Omega_{0} t^{\prime}\right) \nu\left(t^{\prime}\right) \\
f(t) & =-\frac{1}{M \Omega_{0}} \int_{0}^{t} d t^{\prime} \sinh \left(\Omega_{0} t^{\prime}\right) \eta\left(t^{\prime}\right),
\end{aligned}
$$

$\tilde{\Omega}(t)$ renormalizes the natural frequency of the particle, $\gamma(t)$ is the dissipation coefficient, and $D(t)$ and $f(t)$ are the difusion coefficients, which produce the decoherence effects. $\eta(t)$ and $\nu(t)$ are the dissipation and noise kernels, respectively,

$$
\begin{aligned}
& \eta(t)=\int_{0}^{\infty} d \omega I(\omega) \sin \omega t \\
& \nu(t)=\int_{0}^{\infty} d \omega I(\omega) \operatorname{coth} \frac{\beta \omega}{2} \cos \omega t
\end{aligned}
$$

and $I(\omega)$ is the spectral density of the environment. In the high temperature limit of an ohmic environment (where $I(\omega) \propto \omega)$ the coefficients in Eq.(9) become constants. In particular, the diffusion coefficient can be approximated by $D \simeq 2 \gamma_{0} k_{\mathrm{B}} T M$, where $\gamma_{0}$ is the dissipation coefficient [6]. In this limit, while $\gamma_{0}$ is a constant and $D \propto T$, the dissipation coefficient is $f \propto T^{-1}$. Therefore the term proportional to $D$ is the relevant term in the master equation.

Alternatively, one can write an equation of the Fokker-Planck type for the reduced Wigner function [6]. It is given by

$$
\dot{W}_{r}(x, p, t)=-\left\{H_{\mathrm{syst}}-\frac{1}{2} M \tilde{\Omega}^{2}(t), W_{r}\right\}_{\mathrm{PB}}+2 \gamma(t) \partial_{p}\left(p W_{r}\right)+D(t) \partial_{p p}^{2} W_{r}-f(t) \partial_{p x}^{2} W_{r}
$$

where the first term on the right-hand side is a Poisson bracket. 
Let us solve Eqs.(8) and (10) using again a Gaussian ansatz for the reduced density matrix. The adequate generalization of Eq. (3) is

$$
\rho_{r}\left(x, x^{\prime}, t\right)=N(t) e^{-A(t) x^{2}-B(t) x^{2}-C(t) x x^{\prime}},
$$

where $C(t)$ is a real function. The master equation, in the high temperature limit, becomes

$$
\begin{aligned}
\dot{a} & =4 a b-2 \gamma_{0} a+2 \gamma_{0} \tilde{T}+\gamma_{0} C \\
\dot{b} & =-2 a^{2}+2 b^{2}-2 \gamma_{0} b+\frac{1}{2} C^{2}-\frac{1}{2} \tilde{\Omega}^{2} \\
\dot{C} & =4 \gamma_{0} a+4 C b-2 \gamma_{0} C-4 \gamma_{0} \tilde{T} \\
\dot{N} & =2 N b,
\end{aligned}
$$

where we are denoting $\tilde{T}=k_{\mathrm{B}} T$, and we have set $M=1$.

Using again the variables $\Sigma$ and $\Delta$ the reduced density matrix reads

$$
\rho_{r}(\Sigma, \Delta, t)=N(t) e^{-(2 a-C) \Delta^{2}} e^{-(2 a+C) \Sigma^{2}} e^{-4 i b \Sigma \Delta},
$$

while the reduced Wigner function is exactly evaluated as

$$
W_{r}(x, p, t)=\frac{1}{\pi} \sqrt{\frac{2 a+C}{2 a-C}} e^{-(2 a+C) x^{2}} e^{-\frac{(p-2 x b)^{2}}{(2 a-C)}},
$$

From the last two equations we see that the relevant function to describe correlations and decoherence is now $2 a-C$. For $2 a-C=O(1)$ we have both correlations and decoherence. The set of Eqs.(12) can be easily solved numerically. In Fig. 2 we show the behaviour of $2 a-C$ as a function of time. We see that it tends asymptotically to a constant of order one (of course the asymptotic value depends on the properties of the environment).

The main conclusion of this section is the following. In order to study a sudden quench phase transition, at early times we can use the upside down potential. When the system is isolated, due to the high squeezing of the initial wave packet $x$ and $p$ become classically correlated. The density matrix is not diagonal. The "correlation time" depends on the shape of the potential. When the particle is coupled to an environment, a true quantum to classical transition takes place. The Wigner function becomes peaked around a classical trajectory and the density matrix diagonalizes. The decoherence time depend on the diffusion coefficient $D$.

\section{INCLUDING SELF-INTERACTION: VARIATIONAL APPROXIMATIONS}

Let us now consider a more realistic model by adding a $\lambda x^{4}$ term to the Lagrangian of the unstable quantum particle.

Of course the problem no longer admits an exact, analytical solution. Before presenting the numerical solution (Section IV), we would like to discuss some analytical approximations based on the time dependent variational method developed by Jackiw and Kerman [11]. This will be useful to understand the validity of similar approximations in field theory.

The variational method is based on the definition of an "effective action" 


$$
\Gamma=\int d t\left\langle\psi\left|i \frac{\partial}{\partial t}-\hat{H}\right| \psi\right\rangle
$$

When $\Gamma$ is stationary against variations of the state $|\psi\rangle$ (with $\langle\psi \mid \psi\rangle=1$ ), the state satisfies the Schröedinger equation. Approximated solutions are obtained by minimizing the effective action within a family of trial wave functions.

Following the work of Jackiw and Kerman, Cooper et al 12 studied the dynamics of a quantum particle in a double well potential in the so called Gaussian approximation. In this approximation, the problem is equivalent to the inverted oscillator with a self-consistent frequency $\Omega_{\mathrm{sc}}^{2}=\Omega_{0}^{2}-3 \lambda\left\langle x^{2}\right\rangle$.

In order to analyze a system coupled to an environment, one should generalize the variational principle to the density matrix. Although such an extension does exist (see Ref. [13), we follow here an equivalent and simpler method: we replace the renormalized frequency by the self-consistent one $\Omega_{\mathrm{sc}}^{2}=\tilde{\Omega}^{2}-3 \lambda\left\langle x^{2}\right\rangle$ in the master equation. Thus, assuming that the density matrix has the same form given in Eq.(11), the evolution equations for the real functions $a(t), b(t)$, and $C(t)$ can be easily obtained from Eq.(12). The variational equations then read

$$
\begin{aligned}
\dot{a} & =4 a b-2 \gamma_{0} a+2 \gamma_{0} \tilde{T}+\gamma_{0} C \\
\dot{b} & =-2 a^{2}+2 b^{2}-2 \gamma_{0} b+\frac{1}{2} C^{2}-\frac{1}{2} \tilde{\Omega}^{2}+\frac{3}{4} \frac{\lambda}{(2 a+C)} \\
\dot{C} & =4 \gamma_{0} a+4 C b-2 \gamma_{0} C-4 \gamma_{0} \tilde{T} \\
\dot{N} & =2 N b .
\end{aligned}
$$

As before, we are interested in the time dependence of the function $2 a-C$. We have solved numerically Eqs.(16) for different values of the parameters. In Fig. 3 we show the time dependence of $2 a-C$ without environment $\left(\gamma_{0}=0\right.$, which implies $\left.C=0\right)$. As the result is an oscillating function, the width of the Wigner function and the non-diagonal part of the density matrix do oscillate. The self-interacting part of the potential forbids the squeezing of the initial state. Therefore, there are no correlations nor decoherence. When the coupling to the environment is turned on, $2 a-C$ tends to a constant of order one. The environment produces the quantum to classical transition (Fig. 4).

As has been noted by Cooper et al, the Gaussian approximation gives good results up to the time where the non-linearities of the potential can be neglected. To go beyond this point it is necessary to improve the approximation. Cheetham and Copeland [14] had proposed an improvement based on the following trial wave function

$$
\psi(x, t)=\mathcal{N}(t) e^{-i b x^{2}}\left[u_{0}(x, t)+a_{2} u_{2}(x, t)\right],
$$

where

$$
\begin{aligned}
& u_{0}(x, t)=\left[\frac{2 a}{\pi}\right]^{\frac{1}{4}} e^{-a x^{2}} \\
& u_{2}(x, t)=\left[\frac{a}{32 \pi}\right]^{\frac{1}{4}} e^{-a x^{2}}\left[8 a^{2} x^{2}-2\right],
\end{aligned}
$$

where $a$ and $b$ are real time-dependent coefficients; $a_{2}(t)$ is a complex function. The variational equations read 


$$
\begin{aligned}
& \dot{a}=4 a b+\lambda \sqrt{2} \frac{\sin \theta}{16 a R} \\
& \dot{b}=-2 a^{2}+2 b^{2}-1+\frac{7 \lambda}{8 a}+\frac{\lambda \sqrt{2} \cos \theta}{16 a R} \\
& \dot{R}=\lambda \sin \theta \frac{\left(\cos \theta+R^{2} \cos \theta+2 R \sqrt{2}+2 R^{3} \sqrt{2}\right)}{16 a^{2} R} \\
& \dot{\theta}=-4 a-\frac{\lambda\left(4 R^{3} \sqrt{2} \cos \theta+2 R^{3} \cos ^{2} \theta-2 \cos ^{2} \theta+1-6 R \sqrt{2} \cos \theta-11 R^{2}\right)}{32 a^{2} R^{2}},
\end{aligned}
$$

where we have written $a_{2}(t)=R e^{i \theta}$.

In Eq.(17) the zeroth order corresponds to the Gaussian approximation. Including only the first non-trivial term in the expansion in Hermite polynomials, Cheetham and Copeland showed an important improvement in the results [14]. For this "post-Gaussian" form of the wave funcion, we may write the density matrix as

$$
\begin{aligned}
\rho\left(x, x^{\prime}, t\right) & =\mathcal{N}^{2}(t) \sqrt{\frac{2 a}{\pi}} e^{-2 a\left(\Sigma^{2}+\Delta^{2}\right)} e^{-4 i b \Sigma \Delta} \\
& \times\left[1+2 \sqrt{2} a\left(a_{2} x^{\prime 2}+a_{2}^{*} x^{2}\right)-2 \sqrt{2} R e a_{2}+\sqrt{2}\left|a_{2}\right|^{2}\left(2 a x^{2} x^{\prime 2}-x^{2}-x^{\prime 2}+\frac{1}{2 a}\right)\right]
\end{aligned}
$$

which produces a complicated Wigner function

$$
\begin{aligned}
W(x, p, t) & =\frac{\mathcal{N}^{2}(t)}{2 \pi} \sqrt{\frac{2 a}{\pi}} e^{-\frac{(p-2 b x)^{2}}{2 a}} e^{-2 a x^{2}}\left[1+B(t)+D(t) p+E(t) p^{2}+F(t) p^{4}\right. \\
& \left.+H(t) x+I(t) x p+J(t) x p^{3}+K(t) x^{2}+L(t) x^{2} p^{2}+M(t) x^{3} p+P(t) x^{4}\right]
\end{aligned}
$$

where the capital letters are functions of $a_{2}, a_{2}^{*}, a$, and $b$.

The Wigner function is clearly non positive definite. To illustrate this fact, we have solved numerically the variational equations for a Gaussian initial state. In Fig. 5 we show the Wigner function for a time where the wave function is no longer Gaussian. The non-Gaussian shape of the wave function produces a non positive Wigner function.

It is in principle possible to include the environment in the improved version of the Gaussian approximation. However, we will not follow here this possibility. Instead, we will include it in the exact numerical solution to the problem.

\section{INCLUDING SELF-INTERACTION: EXACT NUMERICAL SOLUTION}

In order to get a complete answer about the quantum to classical transition for a double well potential, it is necessary to solve exactly the master equation given in Eq.(8) (adding the $\lambda x^{4}$ term to the Hamiltonian $H_{\text {syst }}$ in the first term of the rhs). The Fokker-Planck equation (10) for the reduced Wigner function has an additional term coming from the non-linearities of the potential,

$$
\begin{aligned}
\dot{W}_{r}(x, p, t)= & -\left\{H_{\mathrm{syst}}-\frac{1}{2} M \tilde{\Omega}^{2}(t), W_{r}\right\}_{\mathrm{PB}}+2 \gamma(t) \partial_{p}\left(p W_{r}\right)+D(t) \partial_{p p}^{2} W_{r} \\
& -f(t) \partial_{p x}^{2} W_{r}-\frac{\lambda}{4} x \partial_{p p p}^{3} W_{r},
\end{aligned}
$$


We have solved numerically this Fokker-Planck equation, in the high temperature limit, for different values of the diffusion coefficient $D$, in order to illustrate its relevance in the quantum to classical transition. We have chosen as initial condition a Gaussian state centered at $x_{0}=p_{0}=0$ with minimal uncertainty $\left(\sigma_{x}^{2}=0.5\right.$ and $\left.\sigma_{p}^{2}=0.5\right)$. The Wigner function is initially positive definite, and different from zero only near the top of the potential. We have set the coupling constant $\lambda=0.01$, the renormalized frequencies $\tilde{\Omega}=\omega_{n}=1$ (we are measuring time in units of $\tilde{\Omega}$ ) and the bare masses also equal to one.

It is illustrative to examine first the exact result when the environment is absent (for this case we have solved numerically the Schrödinger equation). The initially Gaussian Wigner function begins to squeeze in the $x=p$ direction and, before the spinodal time $\left(t_{\mathrm{sp}} \sim 2.3\right)$ it becomes a non-positive function (Fig. 6). During the evolution, the Wigner function covers all the phase space (Fig. 7) and it is clear that it is not possible to consider it as a classical probability distribution. Although we started with a special initial state (Gaussian with minimum uncertainty), the non-linearities of the potential make the Wigner function a non-positive distribution.

Let us now consider a coupling with an environment such that the normal difusion coefficient is $D=0.01$. As expected, the evolution of the Wigner function is similar to the previous one at early times (Figs. 8 and 9). However, as can be seen from Figs. 10 and 11, at long times it becomes positive definite and peaked around the classical phase space.

The effect of the environment is more dramatic for larger values of the diffusion coefficient (see Figs. 12 - 15). For $D=0.1$, the Wigner function is almost positive definite for $t \sim 2 t_{\mathrm{sp}}$ (Fig. 13). In our last example, $D=1$, the quantum to classical transition takes place almost instantaneously, even before the quantum particle pass through the spinodal point (Figs. 15 and 16).

It is interesting to note that, as the difusion coefficient grows, the amplitude of the Wigner function falls down. This is due to the fact that the decoherence increases with $D$. The reduced density matrix diagonalizes. As a consequence, its "Fourier transform", the reduced Wigner function, spreads out.

Our numerical results show explicitly that the existence of the environment is crucial in the quantum to classical transition. The decoherence time depends on the temperature and the coupling between system and environment through the difussion coefficient $D$.

Both the Schrödinger equation for the closed problem and the Fokker-Planck equation (10) were numerically solved using a fourth-order spectral algorithm [15]. Numerical checks included carrying out simulations at different spatial and temporal resolutions.

\section{PHASE TRANSITIONS IN FIELD THEORY}

In any field theory where the classical potential has a local maximum at $\phi=0$, the long wavelength modes of the field are unstable, and grow in time. From these modes it should be possible to identify the classical field that plays the role of order parameter of the transition to the minimum of the potential. The emergence of semiclassical coherent, large amplitude field configurations should be a consequence of time evolution. Therefore, we need a quantum field description of the dynamics, for the early stages of phase ordering and growing of long-wavelength fluctuations, as well as for the classicalization and decoherence of such fluctuations. 
There have been different approaches to this problem in the literature. Many works assume that the field can be split as $\phi(x)=\phi_{0}(t)+\hat{\phi}(x)$, where $\phi_{0}$ is the mean value of the field and $\hat{\phi}$ are the quantum fluctuations [16]. This can be only an approximation to the full problem, since by symmetry arguments it is obvious that the mean value of the field must vanish. One should think of $\phi_{0}(t)$ as the mean value of the field inside one of the domains where the phase transition is taken place. In a more realistic approach, the field is split as [5] $\phi(x)=\phi_{<}(x)+\phi_{>}(x)$, where $\phi_{<}$and $\phi_{>}$describe the short and long-wavelength modes of the field. Hopefully, the effective dynamics of the long-wavelength modes should indicate a quantum to classical transition. A third possibility is to address this problem by analyzing the quantum dynamics of the full field, assuming a vanishing mean value, and check that some of the modes become classical variables during the dynamical evolution.

The main technical complication comes from the fact that, as has been pointed out, the initial growth of the quantum fluctuations is so important that a non-perturbative treatment is unavoidable [17]. For this reason, people have considered the so called Gaussian approximation or, alternatively, the large $N$ limit of $O(N)$ models with spontaneous symmetry breaking. In both approximations one assumes that the wave function associated to the different modes of the quantum field is a Gaussian function, with a self-consistent set of parameters.

For concretness, let us describe the work of Boyanovsky et al [18]. Consider an $O(N)$ field theory

$$
\vec{\Phi}(\vec{x}, t)=\left(\vec{\Phi}_{1}(\vec{x}, t), \vec{\Phi}_{2}(\vec{x}, t), \ldots ., \vec{\Phi}_{N}(\vec{x}, t)\right)
$$

with a potential given by

$$
V[\vec{\Phi}]=\frac{1}{2} m^{2}(t) \vec{\Phi} \cdot \vec{\Phi}+\frac{\lambda}{8 N}[\vec{\Phi} \cdot \vec{\Phi}]^{2}
$$

where $m^{2}(t)$ becomes negative for $t>0$. We will focus on the case in which the initial state is symmetric, i.e. $\langle\Phi\rangle=0$.

The Hamiltonian is

$$
H=\sum_{\vec{k}}\left\{\frac{1}{2} \vec{\Pi}_{\vec{k}} \cdot \vec{\Pi}_{-\vec{k}}+\frac{1}{2} W_{k}^{2}(t) \vec{\Phi}_{\vec{k}} \cdot \vec{\Phi}_{-\vec{k}}\right\},
$$

where $\vec{\Phi}_{\vec{k}}$ is the spatial Fourier transform of the field, and $W_{k}$ is defined as

$$
W_{k}^{2}=m^{2}(t)+k^{2}+\frac{\lambda}{2 N} \int \frac{d^{3} k}{(2 \pi)^{3}}\left\langle\vec{\Phi}_{\vec{k}} \cdot \vec{\Phi}_{-\vec{k}}\right\rangle(t) .
$$

We start with a Gaussian initial state, and we assume that the wave functional will be always Gaussian, describing a pure quantum mechanical state,

$$
\Psi[\vec{\Phi}, t]=\prod_{k}\left\{N_{k}(t) e^{-\frac{A_{k}(t)}{2} \vec{\Phi}_{\vec{k}} \cdot \vec{\Phi}_{-\vec{k}}}\right\}
$$

where $A_{k}(t=0)=W_{k}(t<0)$.

The functional Schrödinger equation gives the following differential equations for $N_{k}$ and $A_{k}$, 


$$
\begin{aligned}
\frac{d}{d t} \ln N_{k}(t) & =-\frac{i}{2} A_{k}(t) \\
i \frac{d A_{k}(t)}{d t} & =A_{k}^{2}(t)-W_{k}^{2}(t) .
\end{aligned}
$$

Introducing the notation

$$
A_{k}(t)=-i \frac{\dot{\phi}_{k}(t)}{\phi_{k}(t)},
$$

the dynamical equation in the $1 / N$ approximation becomes

$$
\ddot{\phi}_{k}(t)+W_{k}^{2}(t) \phi_{k}(t)=0 \text {. }
$$

The expectation value of $\vec{\Phi}_{k}(t)^{2}$ in the state of Eq.(27) is given by

$$
\left\langle\vec{\Phi}_{\vec{k}} \cdot \vec{\Phi}_{-\vec{k}}\right\rangle(t)=N\left|\phi_{k}(t)\right|^{2} .
$$

Therefore we have a self-consistent system for $t>0$ given by

$$
\begin{aligned}
& \ddot{\phi}_{k}(t)+\left[k^{2}+M^{2}(t)\right] \phi_{k}(t)=0 \\
& M^{2}(t)=-\mu^{2}+\frac{\lambda}{2} \int \frac{d^{3} k}{(2 \pi)^{3}}\left|\phi_{k}(t)\right|^{2}
\end{aligned}
$$

The numerical solution of these equations reveals the following picture: at early times, the long-wavelength fluctuations "see" an inverted oscillator potential, and grow exponentially in time. This is a linear regime where the self-interaction can be neglected. At intermediate times the backreaction of these fluctuations is as important as the classical terms in the Lagrangian. This period is highly non-linear. At long times times the effective mass of the fluctuations vanishes asymptotically [19].

It can be seen from the wave functional Eq.(27) that, asymptotically, the long-wavelength modes become classically correlated. This is analogous to the situation described by the inverted harmonic oscillator. When $M^{2} \sim 0$, the mode equations (32) can be easily solved 20

$$
\phi_{k}=a \cos k t+b \frac{\sin k t}{k} .
$$

As a consequence, the width of the Gaussian wave function for the mode $k$ increases linearly with time $R e A_{k}{ }^{-1} \sim t$, when $k t<<1$. As we have seen, a Gaussian wave function has a positive definite Wigner function associated to it. Moreover, as the width of the wave function (or the density matrix) increases, the Wigner function becomes sharply peaked around the classical trajectory. This is indeed what happens for $k<<1 / t$. These modes become classically correlated.

In view of the results in the previous sections of this paper, we see that in the large $N$ limit, and at long times, the dynamical evolution of the $O(N)$ model shows "classical correlations" but not a full quantum to classical transition. As for the inverted oscillator 
without environment, the density matrix does not become diagonal. The "correlation time" depends only on the details of the classical potential. I

On the other hand, these classical correlations depend crucially on the Gaussian form of the wave function. To illustrate this point, we consider another example from non relativistic quantum mechanics, the d-dimensional quantum roll, which corresponds to the zero space dimensional limit of the $O(d)$ field theory.

The model is described by the Hamiltonian [21]

$$
H=-\frac{1}{2} \nabla^{2}+V(r)
$$

where

$$
\begin{aligned}
\nabla^{2} & =\sum_{i=1}^{N} \frac{\partial^{2}}{\partial x_{i}^{2}}, \quad r^{2}=\sum_{i=1}^{N} x_{i}^{2} \\
V(r) & =\frac{\lambda}{8 d}\left(r^{2}-r_{0}^{2}\right)^{2} .
\end{aligned}
$$

The Schrödinger equation reads

$$
H \Psi\left(x_{i}, t\right)=i \frac{\partial \Psi\left(x_{i}, t\right)}{\partial t}
$$

This problem can be studied in a multidimensional coordinate system with $r$ the radial coordinate and a set of $d-1$ angular coordinates, such that the Laplacian is

$$
\nabla^{2}=\frac{\partial^{2}}{\partial r^{2}}+\frac{(d-1)}{r} \frac{\partial}{\partial r}-\frac{L_{d-1}^{2}}{r^{2}} .
$$

Here $L_{d-1}^{2}$ is the generalized orbital angular momentum operator. Starting with a Gaussian, radially symmetric initial state centered at the top of the hill $(r=0)$, there angular momentum will vanish. Therefore the Schrödinger equation can be re-written as

$$
\tilde{H}(r, l) \Phi(r, t)=i \frac{\partial \Phi(r, t)}{\partial t}
$$

where

$$
\begin{aligned}
\Phi(r, t) & =r^{\frac{(d-1)}{2}} \Psi(r, t) \\
\tilde{H} & =-\frac{1}{2} \frac{\partial^{2}}{\partial r^{2}}+U(r) \\
U(r) & =\frac{(d-1)(d-3)}{8 r^{2}}+\frac{\lambda}{8 d}\left(r^{2}-r_{0}^{2}\right)^{2} .
\end{aligned}
$$

\footnotetext{
${ }^{1}$ There is a quantitative difference with the example we presented in Section II: there the width of the wave function increased exponentially. Here, as the mode become massless, the growth of the width is only linear in time (an inverted oscillator would correspond to a negative mass).
} 
Following Mihiala et al [21], we have solved the Schrödinger equation numerically. We took an initial Gaussian state centered at the potential top, and used $d=20, r_{0}=6.4$, and $\lambda=10$. In Fig. 17 we plot $\left\langle r^{2} / d>\right.$. After a period of oscillations, this function reachs a constant value for large times. This asymptotic value would imply a vanishing effective mass in the large $d$ approximation. However, as can be seen from Fig. 18, the Wigner function is not positive definite even when the effective mass vanishes. The reason for this is that the wave function that describes the d-dimensional slow roll has a complicated structure, and cannot be approximated by a Gaussian function (see Fig. 19).

The lesson we learned from this example is that, in order to get a Wigner function that is positive definite and peaked around a classical trajectory at long times, it is necessary to have both vanishing effective mass and a Gaussian wave function. Therefore, it is quite possible that in a field theory calculation with finite $N$ there will be no classical limit nor classical correlations unless one considers the coupling of the field with an environment. The presence of the environment will introduce a new temporal scale, the decoherence time, which will indicate when the order parameter becomes a classical variable. For each mode, the decoherence time could be shorter than the spinodal time, allowing for a classical description of these modes in the non-linear regime. A realistic treatment of the environment seems to be crucial to understand the classical limit in field theory phase transitions.

\section{ACKNOWLEDGMENTS}

This work was supported by Universidad de Buenos Aires, Conicet (Argentina) and Fundacion Antorchas. 


\section{REFERENCES}

[1] See for example, Decoherence and the appearance of a classical world in quantum theory, D. Giulini et al, Springer Verlag (1996); K. Molmer, Phys. Rev. A55, 3195 (1997); J. Mod. Optics 44, 1937 (1997)

[2] W.G. Unruh and W.H. Zurek, Phys. Rev. D40, 1071 (1989)

[3] W.H. Zurek, Nature 317, 505 (1985); Phys. Rep. 4, 276 (1996) and references therein

[4] G. Karra and R.J. Rivers, Phys. Lett. B414, 28 (1997); R.J. Rivers, 3rd Colloque Cosmologie, Observatoire de Paris, in the Proceedings edited by H.J. de Vega and N. Sánchez, 314, World Scientific (1995); A.J. Gill and R.J. Rivers, Phys. Rev. D51, 6949 (1995); G.J. Cheetham, E.J. Copeland, T.S. Evans, and R.J. Rivers, Phys. Rev. D 47, 5316 (1993); G.J Stephens, E.A. Calzetta, B.L. Hu, and S. Ramsey, Phys. Rev. D59, 045009 (1999)

[5] F.C. Lombardo and F.D. Mazzitelli, Phys. Rev. D53, 2001 (1996); see also C. Greiner and B. Muller, Phys. Rev. D55, 1026 (1997)

[6] B.L. Hu, J.P. Paz, and Y. Zhang, Phys. Rev. D45, 2843 (1993); D47, 1576 (1993); J.P. Paz, S. Habib, and W.H. Zurek, Phys. Rev. D47, 488 (1993)

[7] A. Guth and S.Y. Pi, Phys. Rev D32, 1899 (1985)

[8] R. Laflamme and J. Louko, Phys. Rev. D43, 3317 (1991)

[9] W.H. Zurek and J.P. Paz, Phys. Rev. Lett. 72, 2508 (1994)

[10] C. Kiefer, J. Lesgourgues, D. Polarski, and A.A. Starobinsky, Class. Quant. Grav. 15, L67 (1998); C. Kiefer, D. Polarski, and A.A. Starobinsky, gr-qc/9910065 and references therein

[11] R. Jackiw and A. Kerman Phys. Lett. 71A, 158 (1979)

[12] F. Cooper, S.Y. Pi, and P. Stancioff, Phys. Rev D34, 3831 (1986)

[13] R. Balian and R. Veneroni, Phys. Rev. Lett. 47, 1353 (1981)

[14] G.J. Cheetham and E.J. Copeland, Phys. Rev. D53, 4125 (1996)

[15] M. Feit, J. A. Fleck Jr., and A. Steiger, J. Comp. Phys. 47, 412 (1982)

[16] J.P. Paz and F.D. Mazzitelli, Phys. Rev. D37, 2170 (1988); F.D. Mazzitelli and J.P. Paz, Phys. Rev. D39, 2234 (1989); S.A. Ramsey and B.L. Hu, Phys. Rev. D56, 661 (1997); D. Boyanovsky and H.J. de Vega, Phys. Rev.D47, 2343 (1993); M. Gleiser and R. Ramos, Phys. Rev D50, 2441 (1994)

[17] F. Cooper, S. Habib, Y. Kluger, E. Mottola, J.P. Paz, and P. R. Anderson, Phys. Rev. D50, 2848 (1994); F. Cooper, Y. Kluger, E. Mottola, and J.P. Paz, Phys. Rev. D51, 2377 (1995); D. Boyanovsky and H.J. de Vega, in Ref. [16]

[18] D. Boyanovsky, H.J. de Vega, and R. Holman, Phys. Rev. D49, 2769 (1994); D. Boyanovsky, H.J. de Vega, R. Holman, D.-S. Lee, and A. Singh, Phys. Rev. D51, 4419 (1995); D. Boyanovsky, D. Cormier, H.J. de Vega, R. Holman, and S. Prem Kumar, Phys. Rev. D57, 2166 (1998)

[19] D. Boyanovsky, C. Destri, H.J. de Vega, R. Holman, and J. Salgado, Phys. Rev. D57, 7388 (1998)

[20] D. Boyanovsky, H.J. de Vega, and R. Holman, Non-equilibrium phase transitions in condensed matter and cosmology: spinodal decomposition, condensates and defects; in Lectures delivered at the NATO Advanced Study Institute: Topological Defects and the Non-Equilibrium Dynamics of Symmetry Breaking Phase Transition; hep-ph/9909372 
[21] B. Mihaila, J. Dawson, F. Cooper, M. Brewster, and S. Habib, The quantum roll in d-dimensions and the large-d expansion; hep-ph/9808234 


\section{Figure Captions}

Figure 1: Function $2 a$ which gives the width of the Wigner function for the upside down harmonic potential.

Figure 2: Width of the Wigner function when an environment is taking into account. We considered an underdamped case with $\gamma_{0}=0.001$ and $\tilde{T}=500$.

Figure 3: Time dependent width of the Wigner function for a self-interacting $\left(\lambda x^{4}\right)$ potential, using the Gaussian approximation.

Figure 4: Same as figure 3 but including an underdamped $\left(\gamma_{0}=0.001\right)$ environment with $\tilde{T}=50$.

Figure 5: Wigner function for the post-Gaussian approximation. It is evaluated at a time $(t=20)$ when the wave function is no longer a Gaussian.

Figure 6: Wigner function for $t=2<t_{\mathrm{sp}}=2.3$, no environment is considered.

Figure 7: Wigner function for $t=4$. As the function is not positive definite, there is no correlation.

Figure 8: Wigner function for $t=2$ including an environment with diffusion coefficient $D=0.01$.

Figure 9: Same Wigner function for time $t=4$. The Wigner function is not positive definite.

Figure 10: Same Wigner function for $t=10$.

Figure 11: Same Wigner function for $t=15$. Only for $t>t_{\mathrm{sp}}$ we have classical correlations and a positive Wigner function.

Figure 12: Early time $(t=2)$ Wigner function for the case $D=0.1$.

Figure 13: Same as figure 12, for $t=4$.

Figure 14: Long time $(t=10)$ behaviour of the Wigner function for $D=0.1$. Here we can consider the system as classical.

Figure 15: Early time $(t=1)$ Wigner function for a diffusion coefficient $D=1$.

Figure 16: Same as figure 15 for $t=2$. Classicality emerges before the spinodal time.

Figure 17: d-dimensional quantum roll. $\left\langle r^{2} / d\right\rangle$ for $d=20, r_{0}=6.4$, and $\lambda=10$.

Figure 18: Wigner function for the d-dimensional example, evaluated at a time $(t=40)$ when the "effective mass" is zero.

Figure 19: $|\phi(r, t)|^{2}$ for the d-dimensional example. The wave function is clearly non Gaussian. 


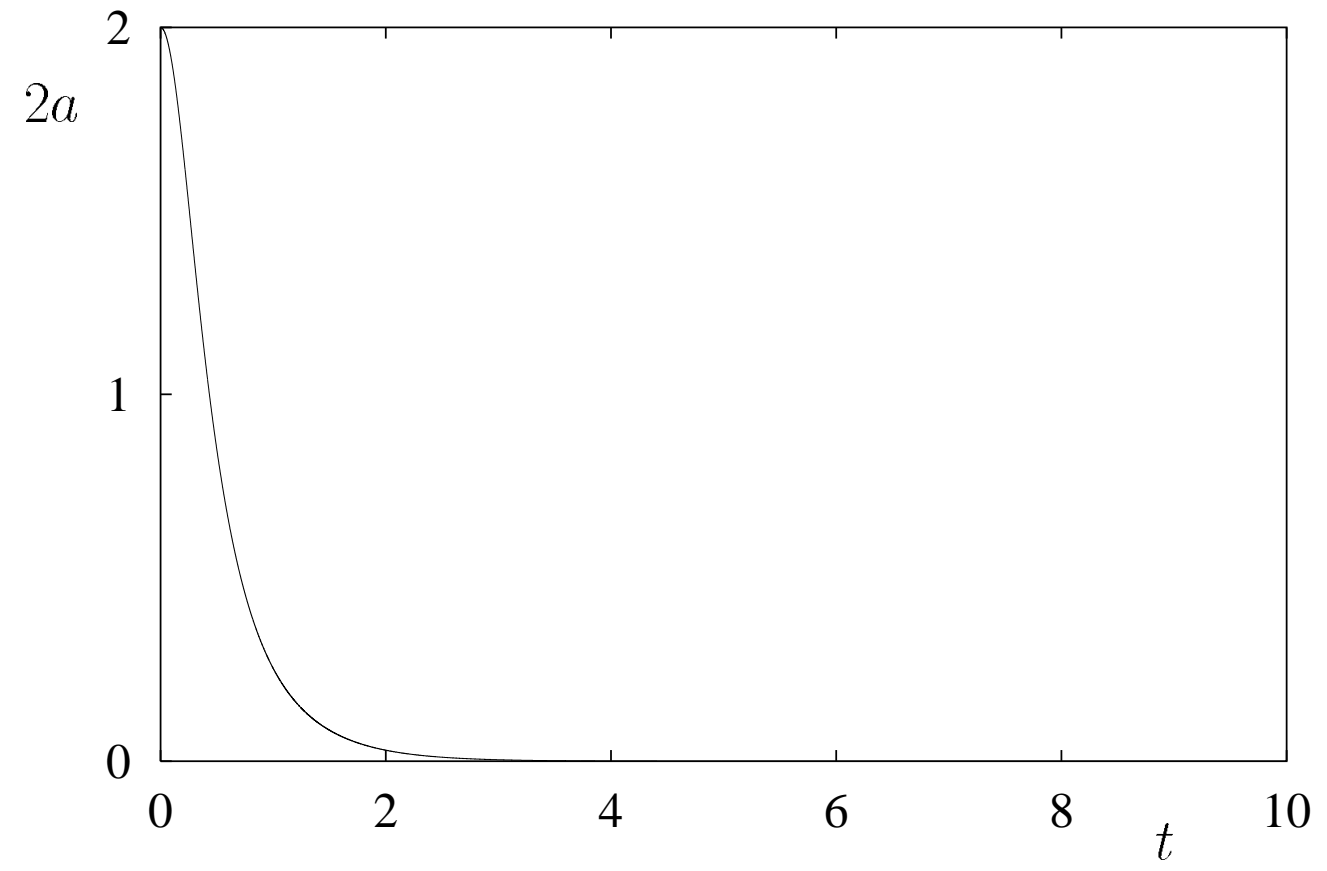

Fig. 1 


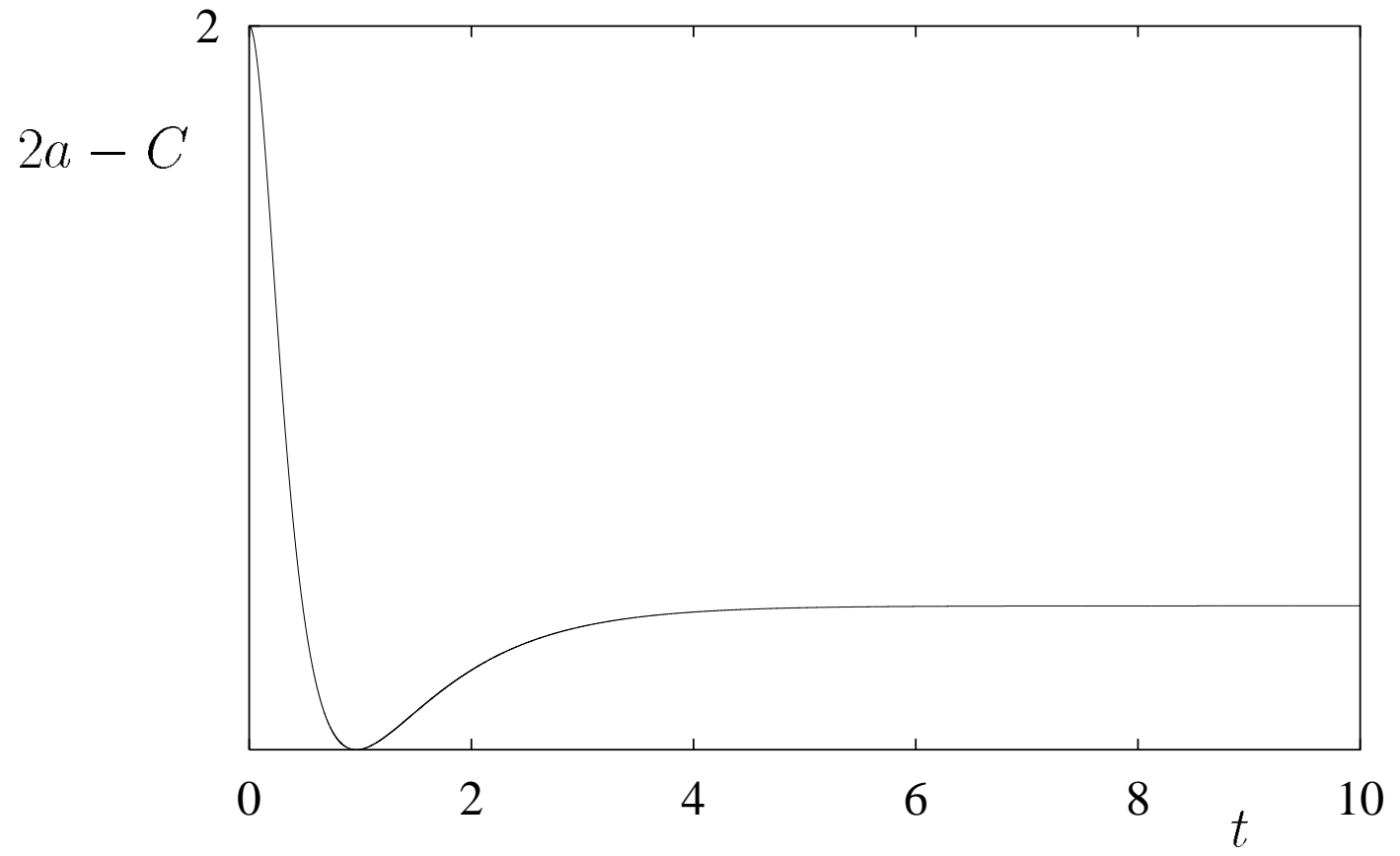

Fig. 2 


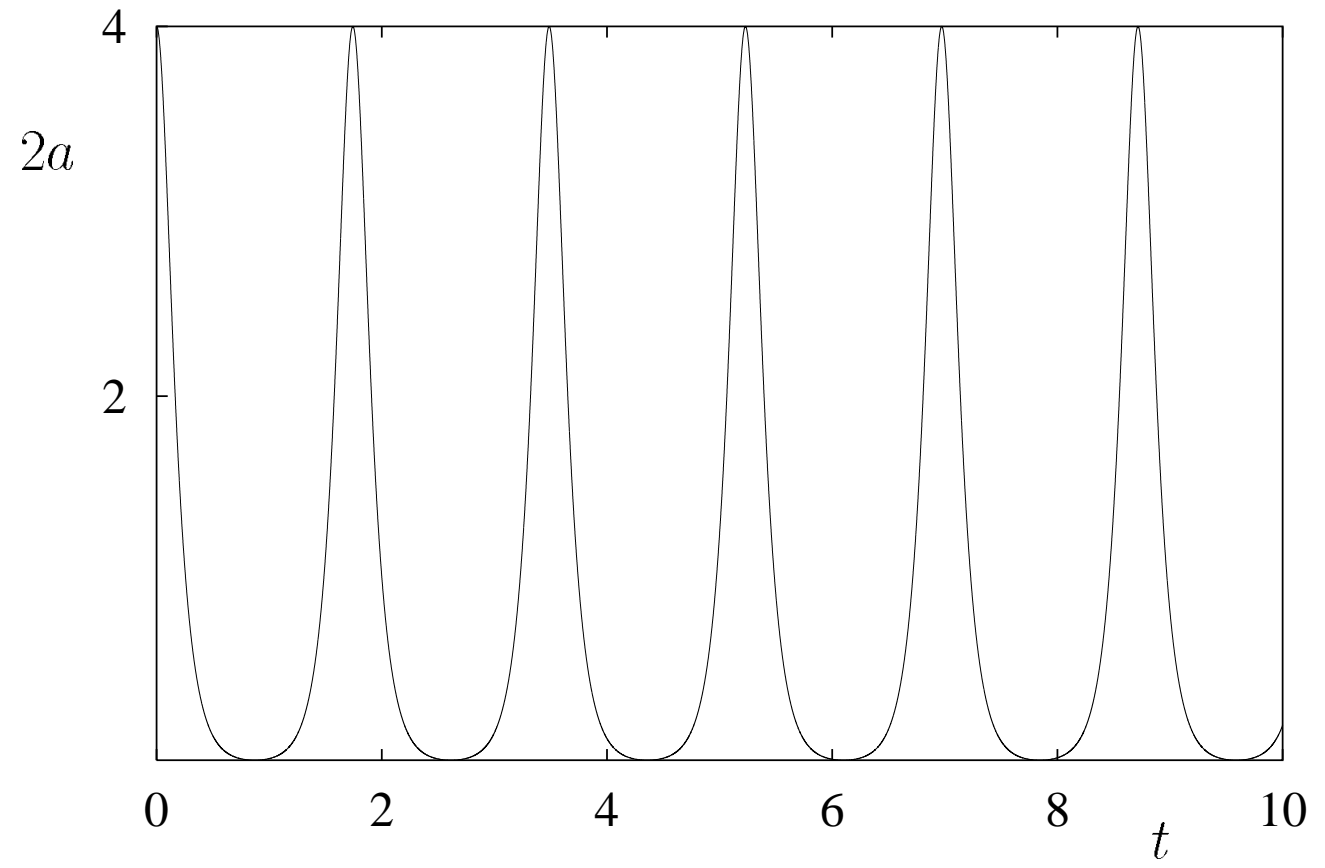

Fig. 3 


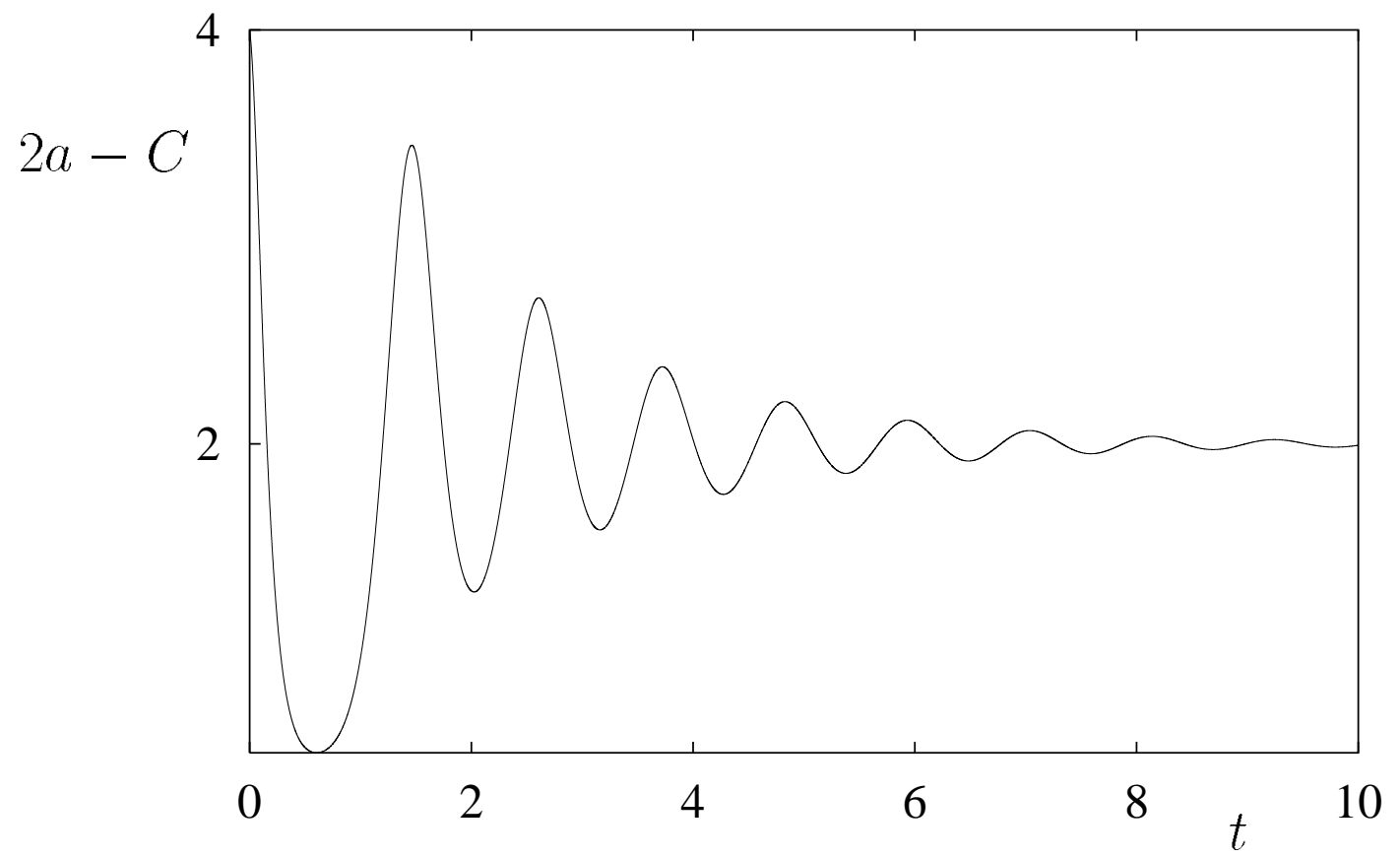

Fig. 4 


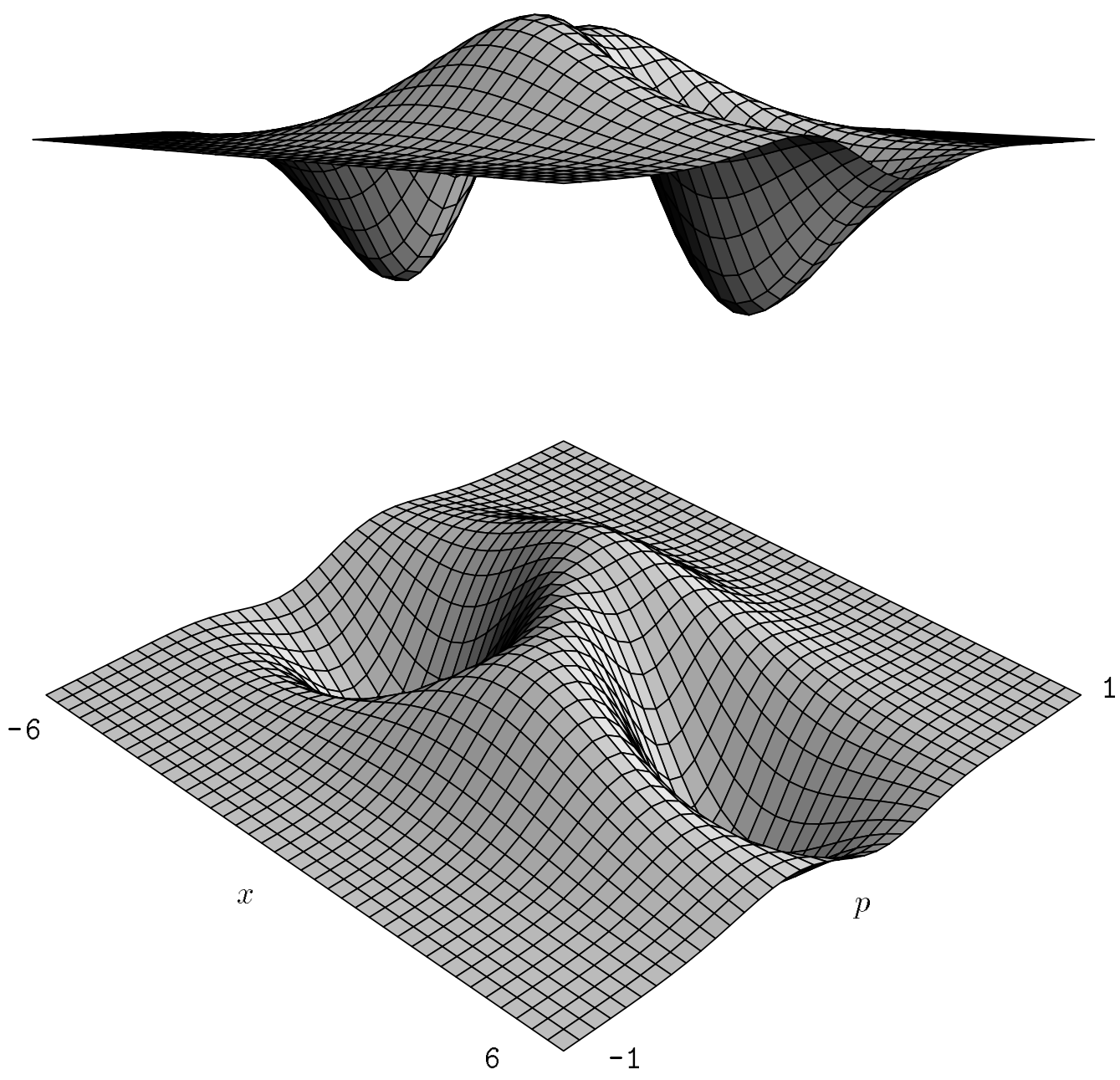

Fig. 5 


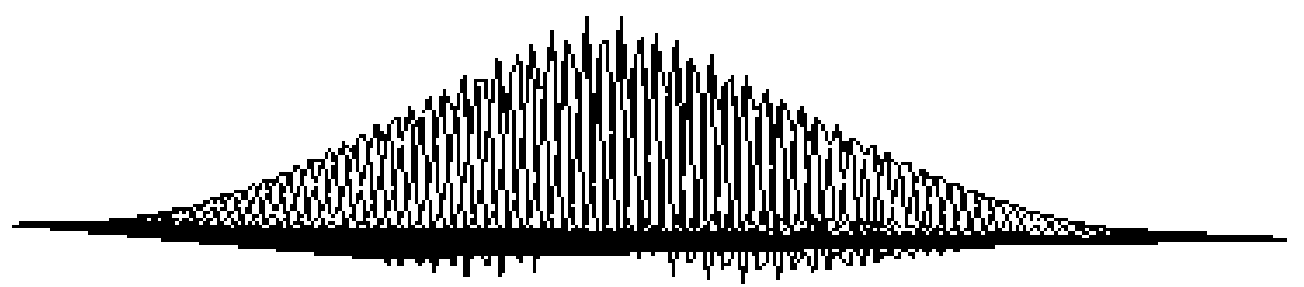

$$
t=2
$$

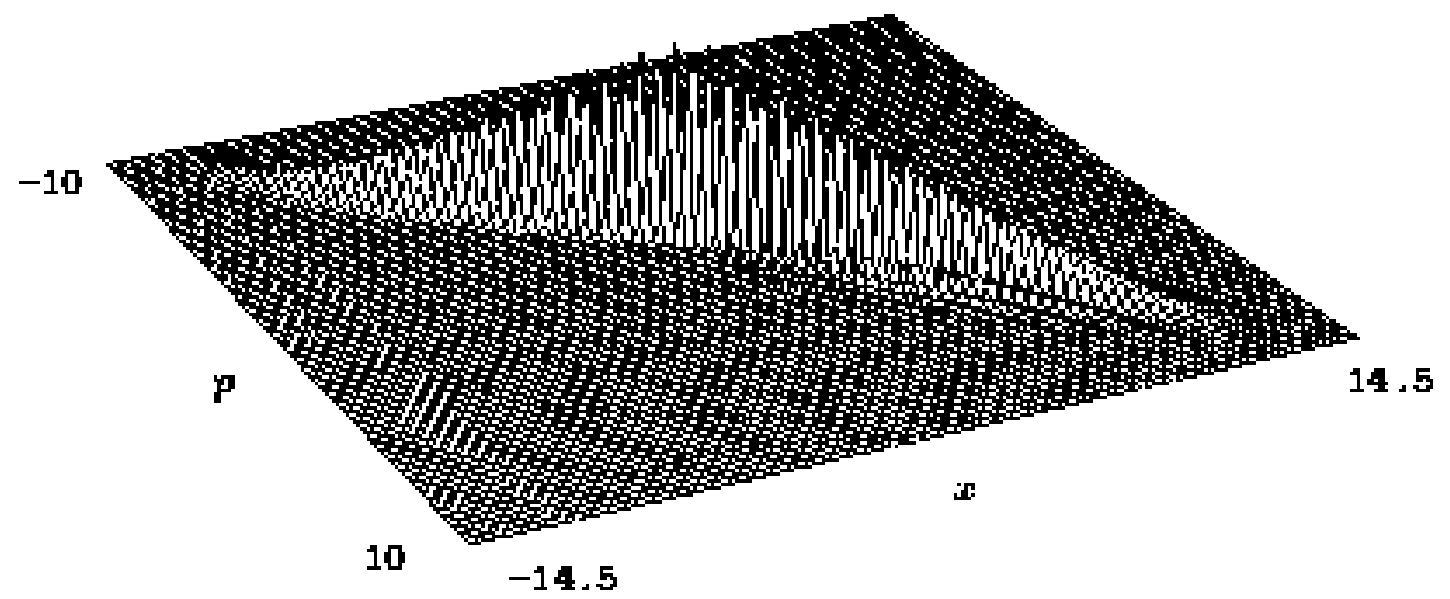

Fig. 6 


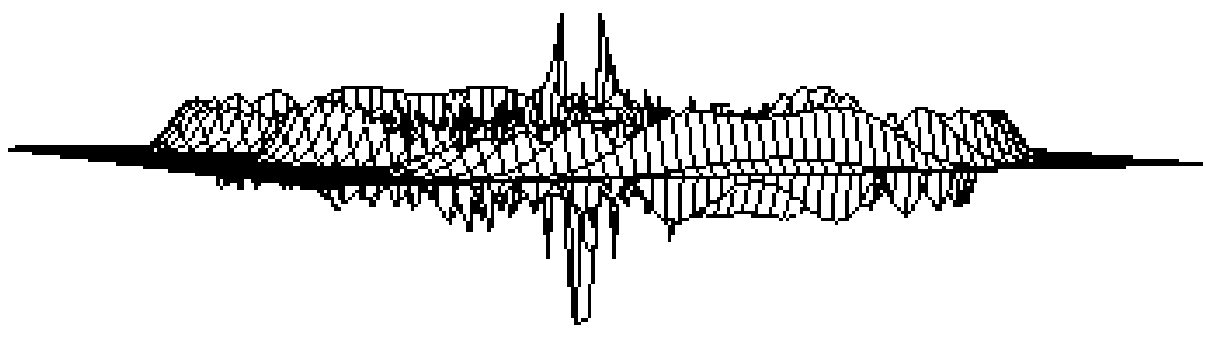

$$
t=4
$$

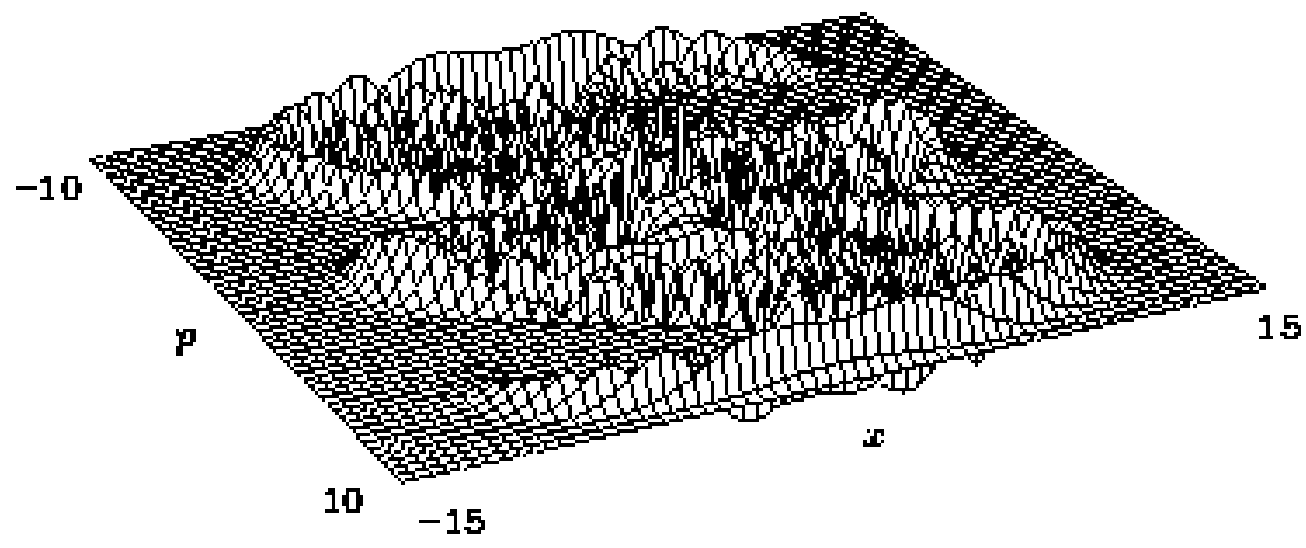

Fig. 7 

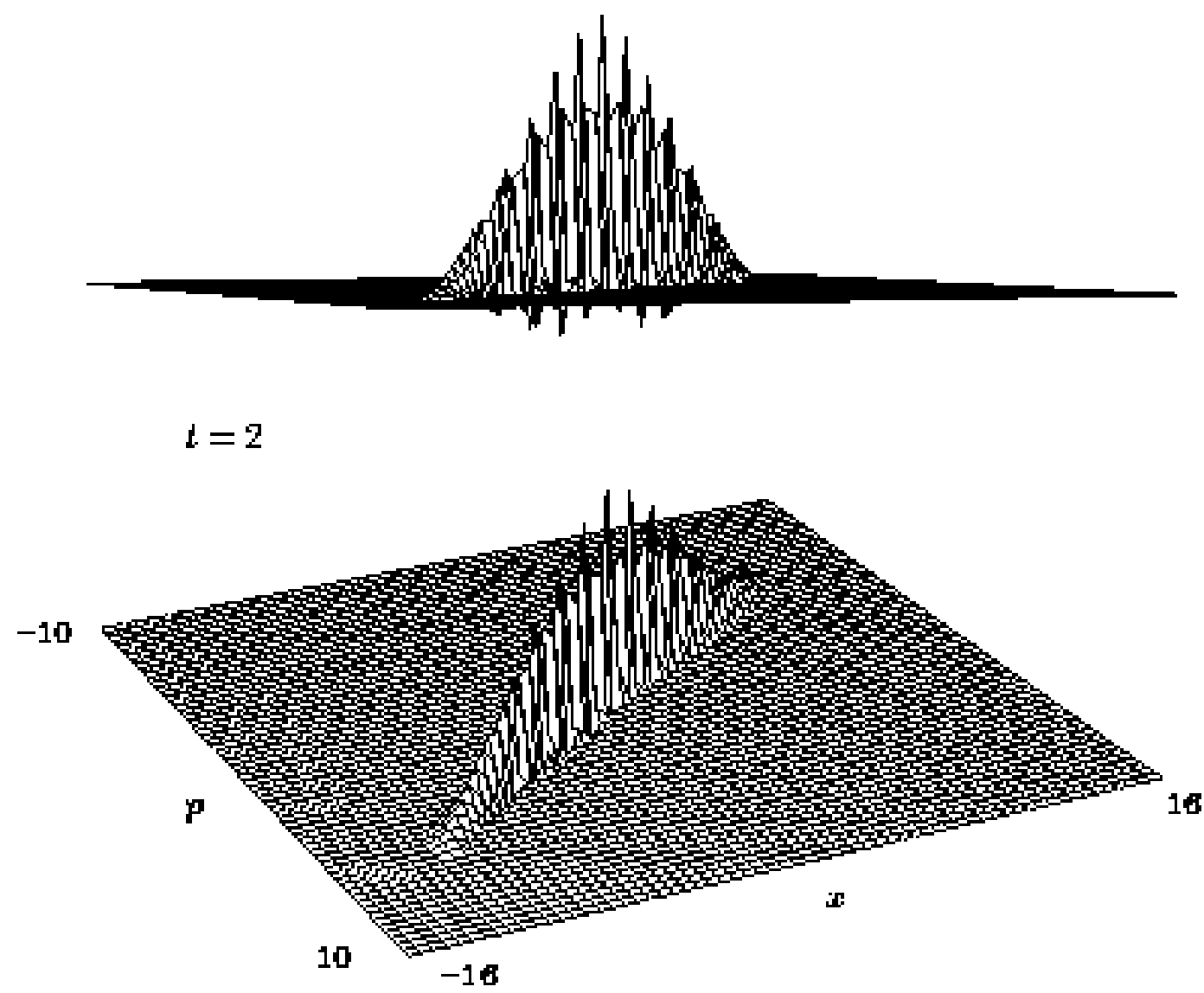

Fig. 8 

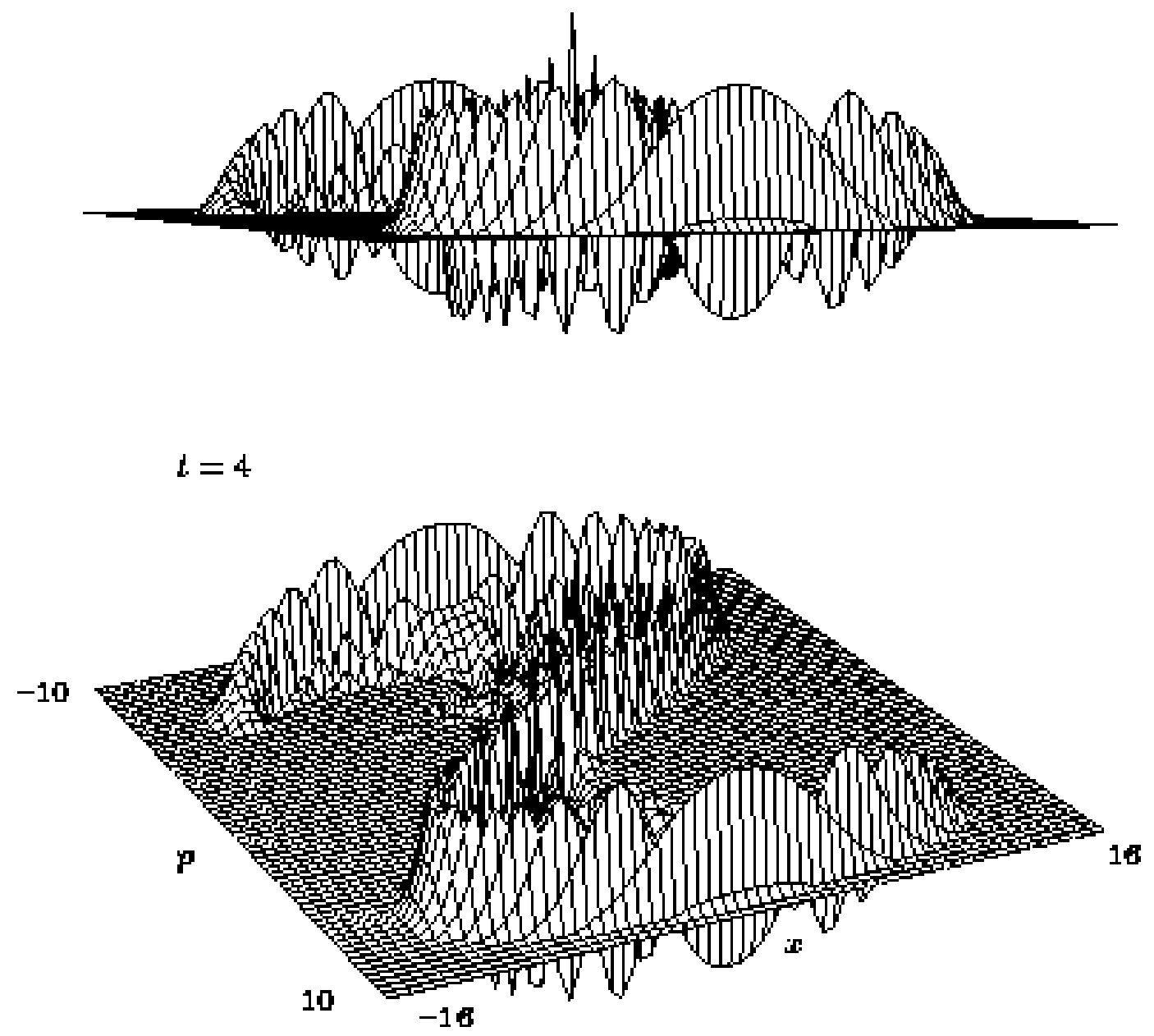

Fig. $\quad 9$ 


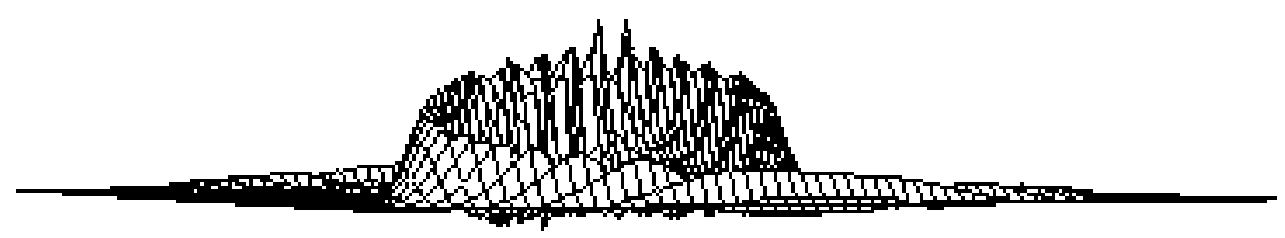

$$
t=10
$$

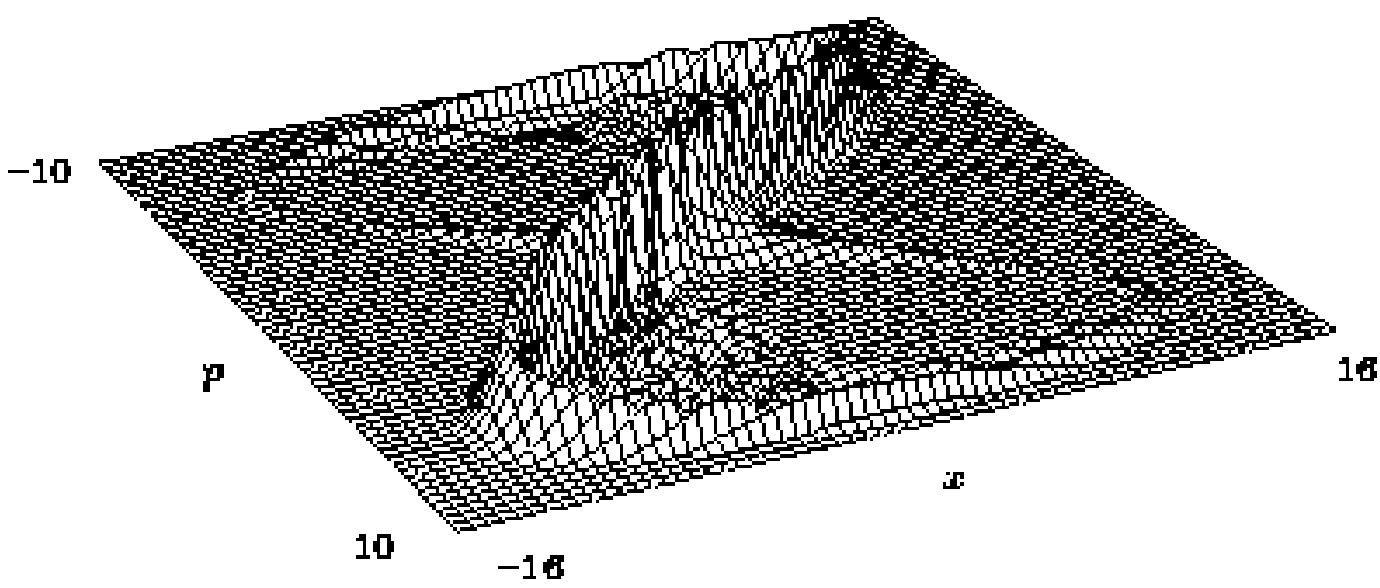

Fig. $\quad 10$ 


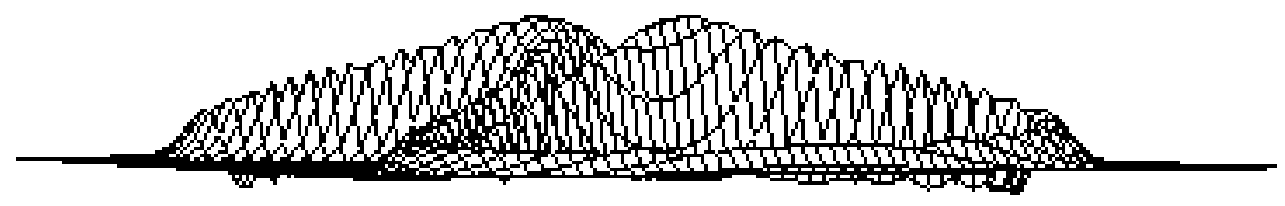

$$
t=15
$$

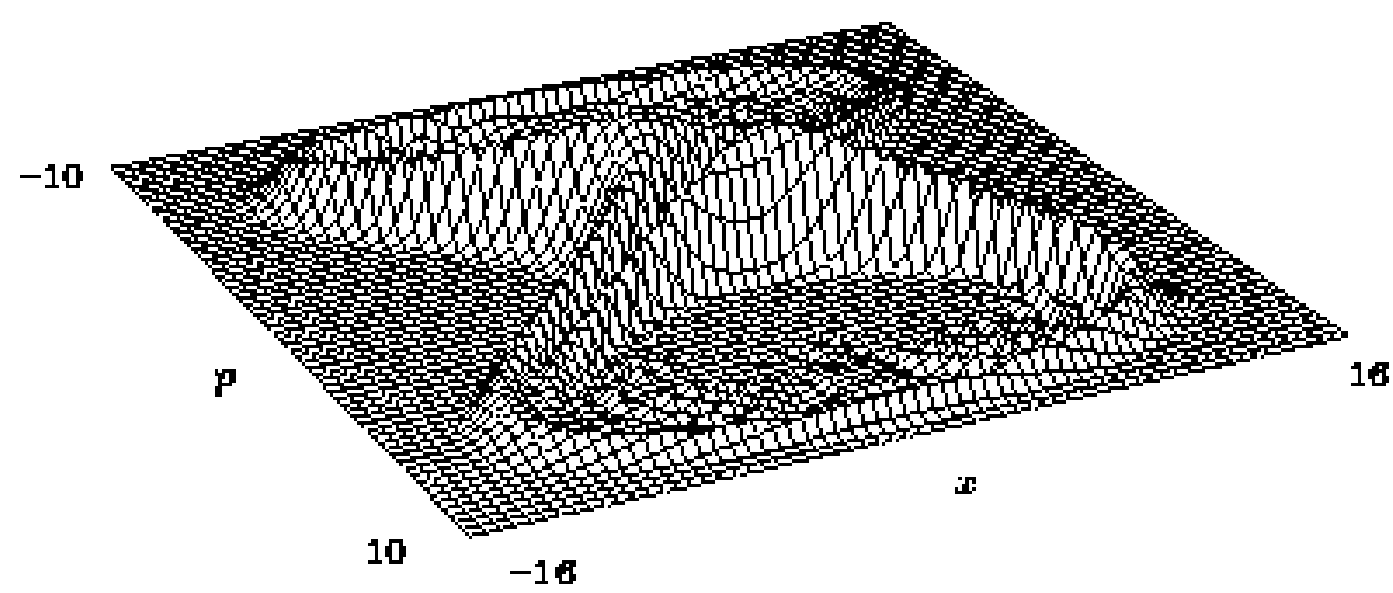

Fig. $\quad 11$ 

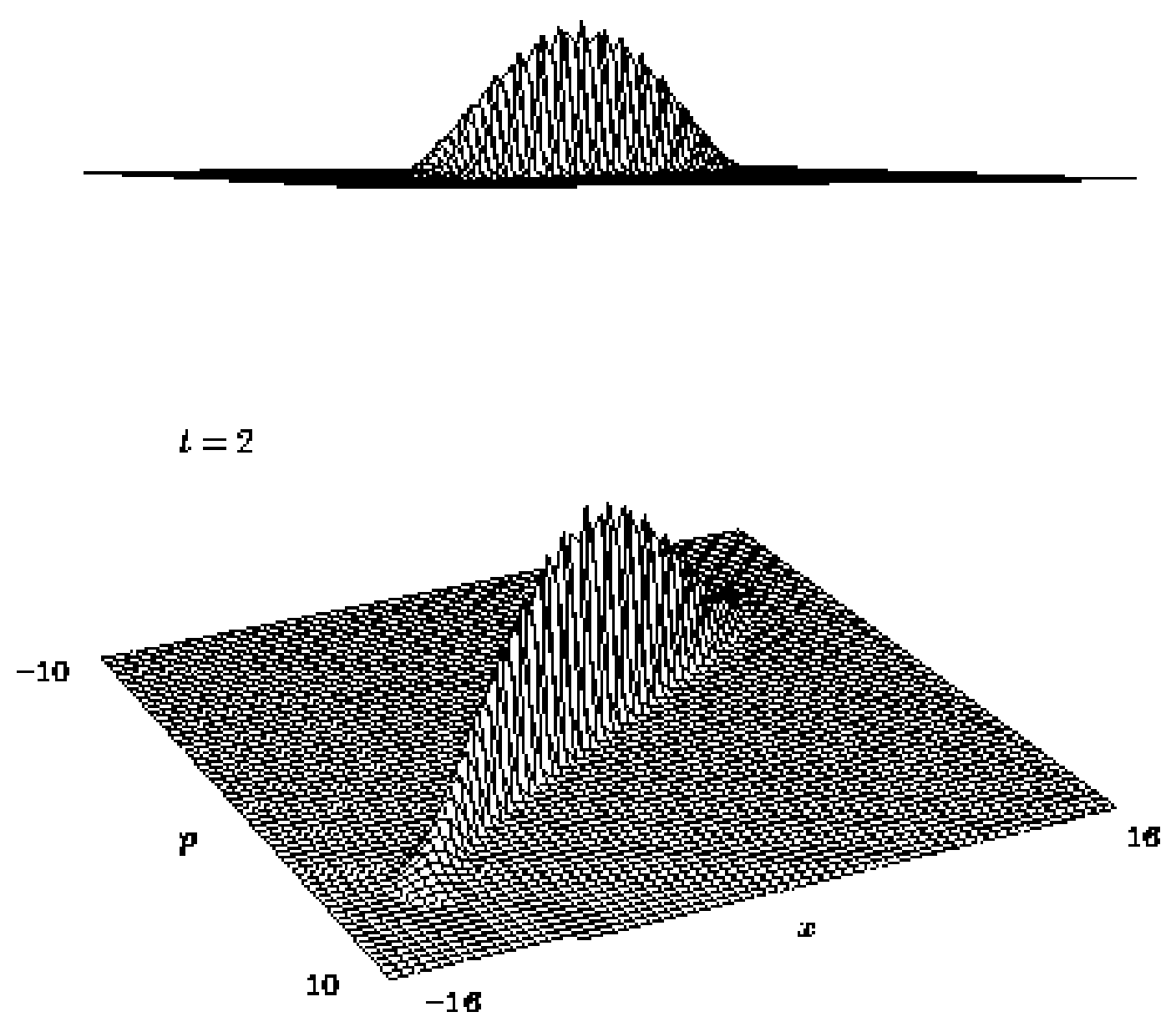

Fig. $\quad 12$ 

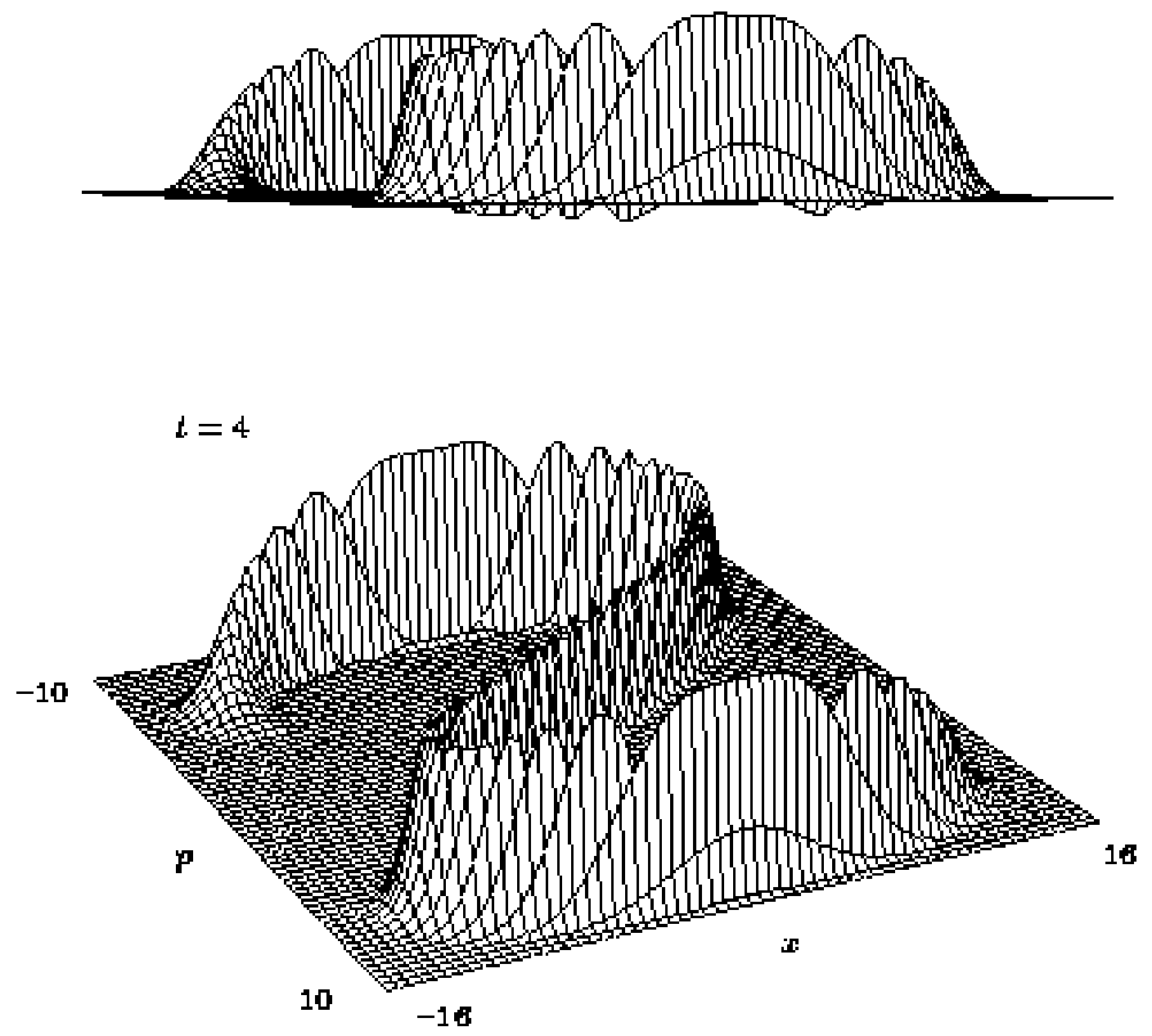

Fig. 13 

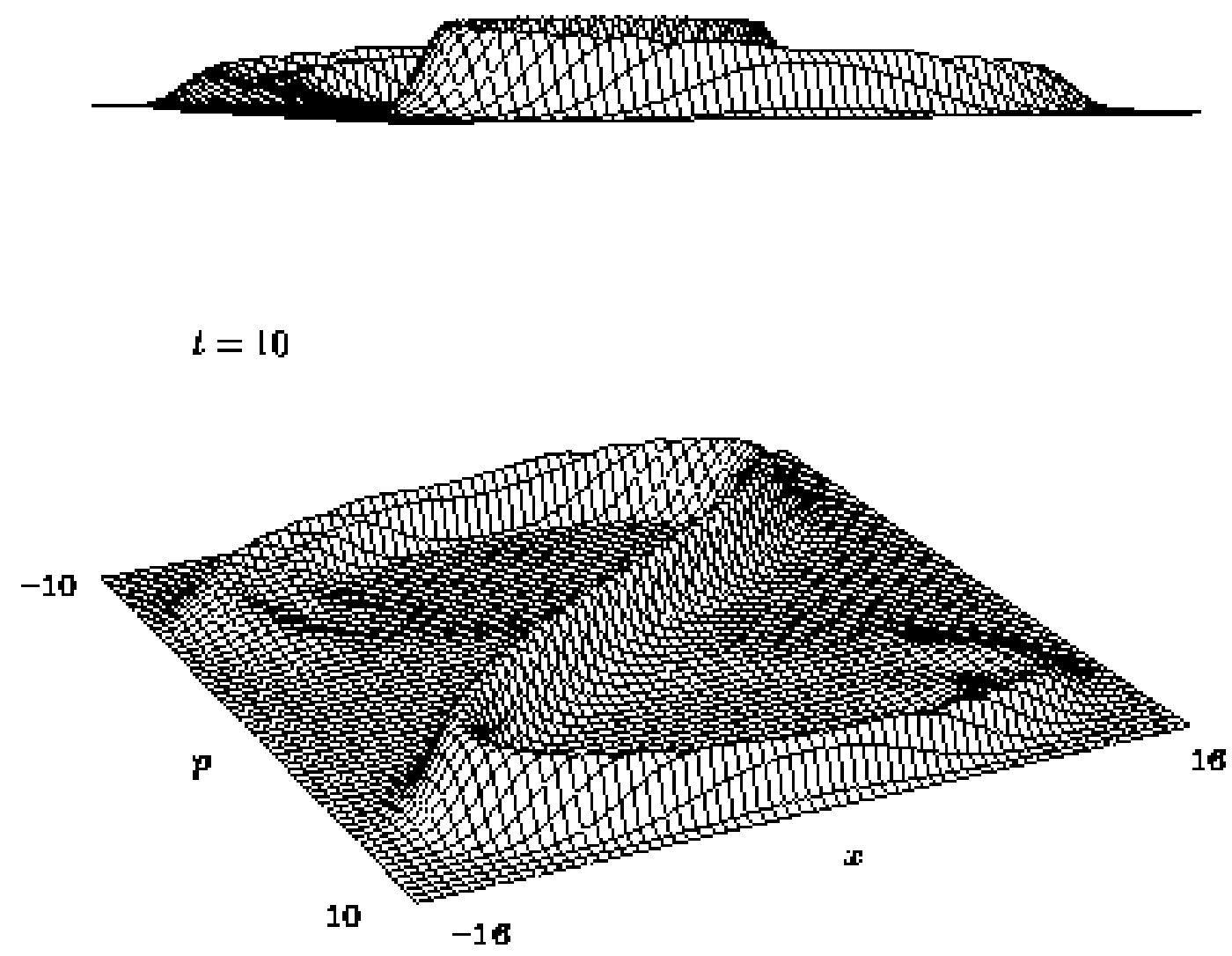

Fig. $\quad 14$ 

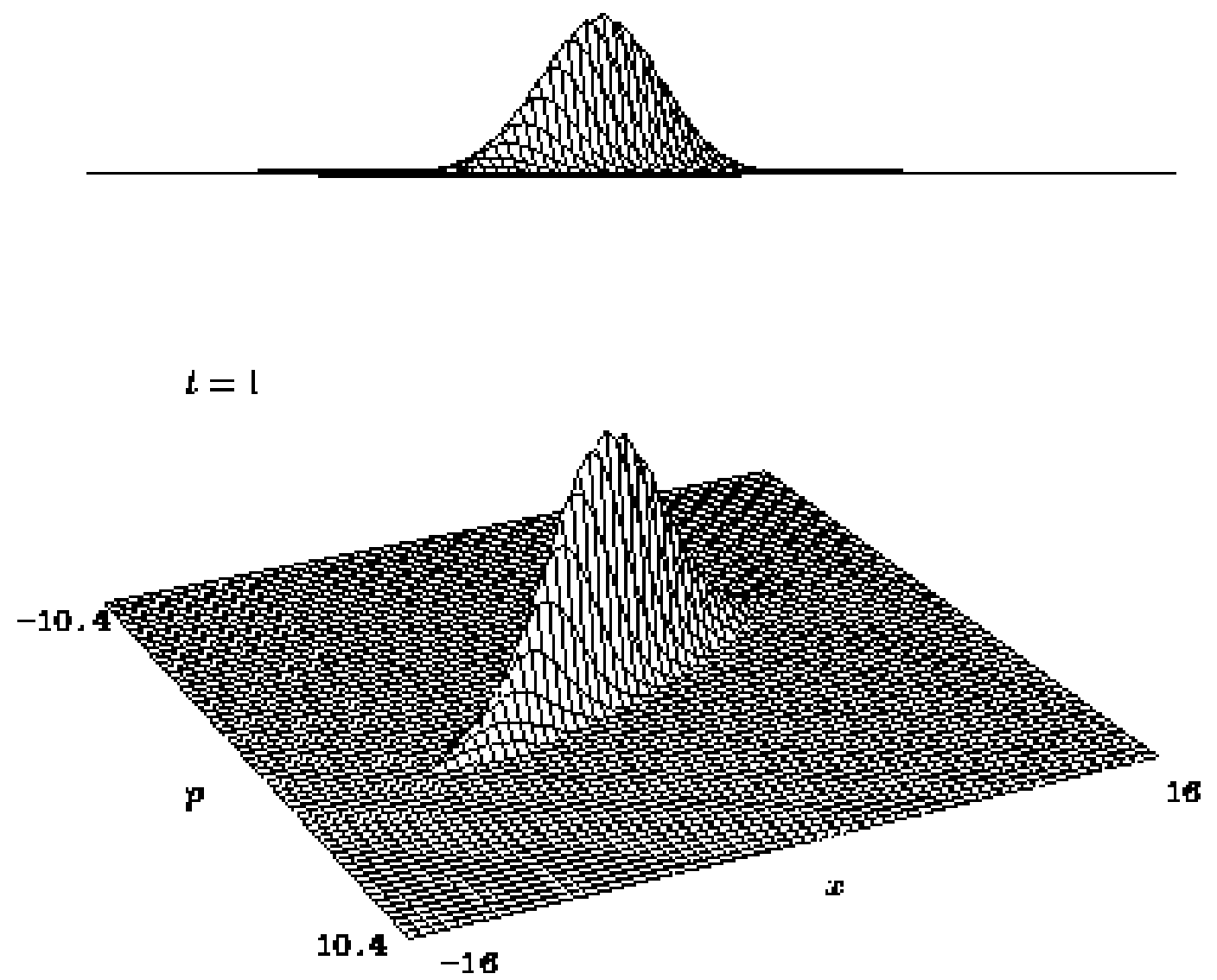

Fig. 15 


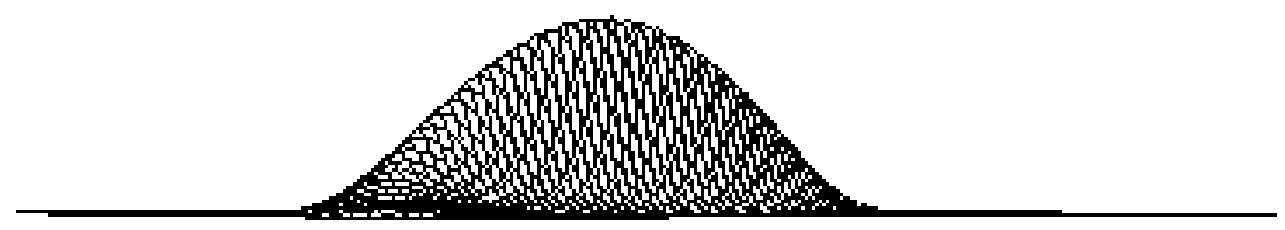

$$
t=2
$$

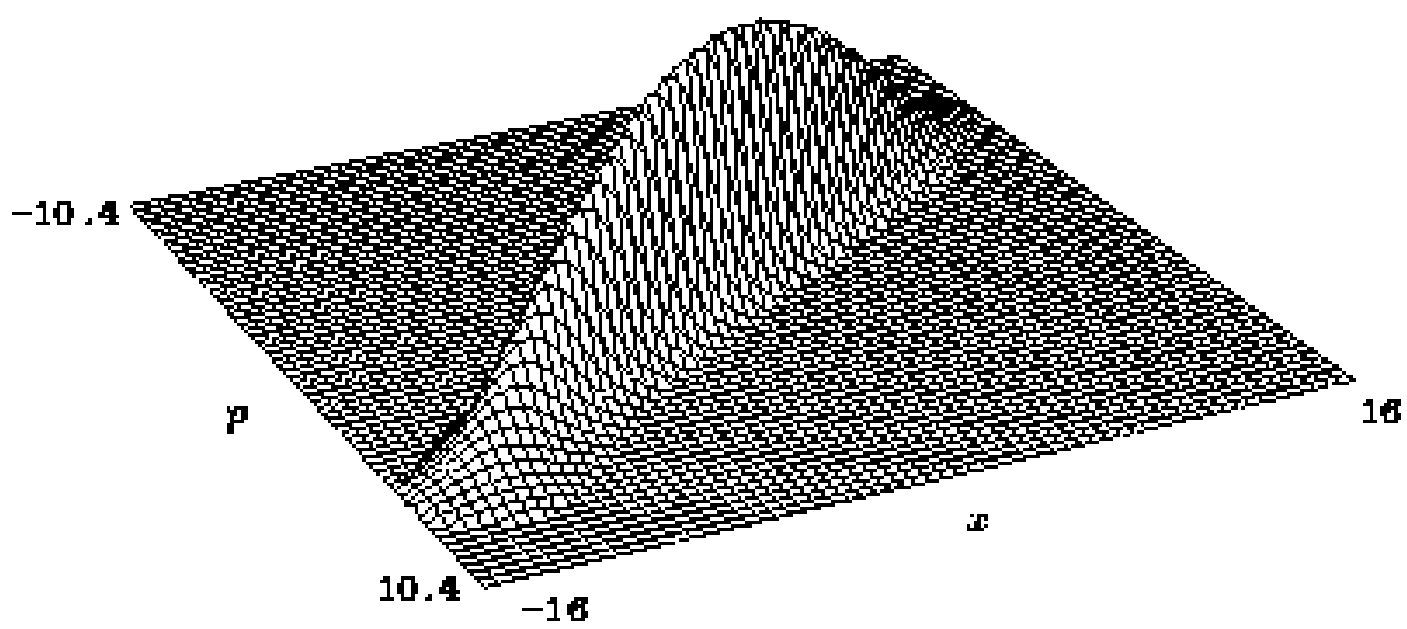

Fig. $\quad 16$ 


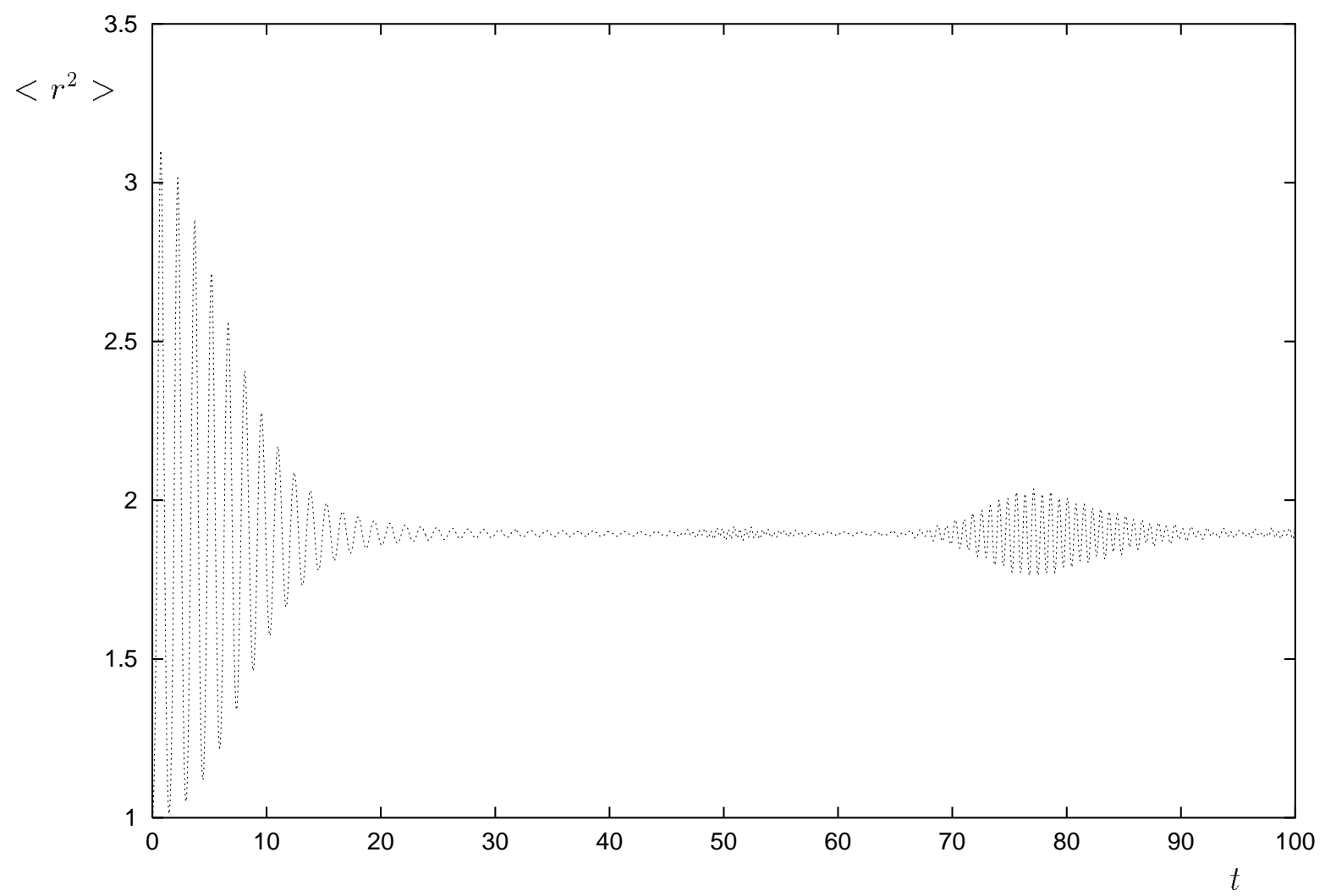

Fig. 17 


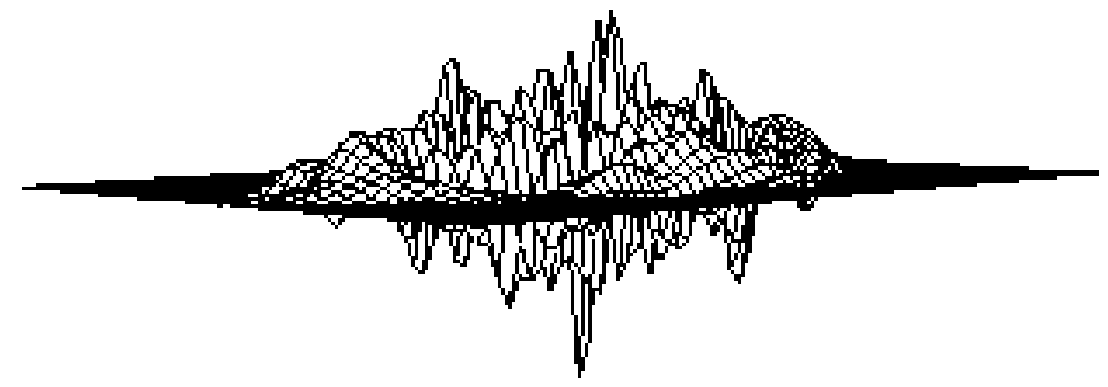

$$
t=40
$$

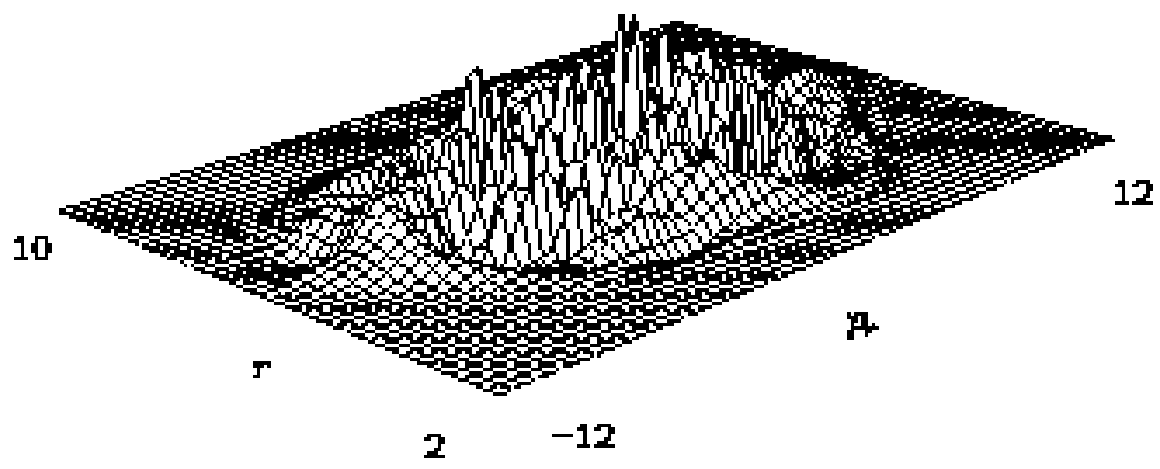

Fig. $\quad 18$ 


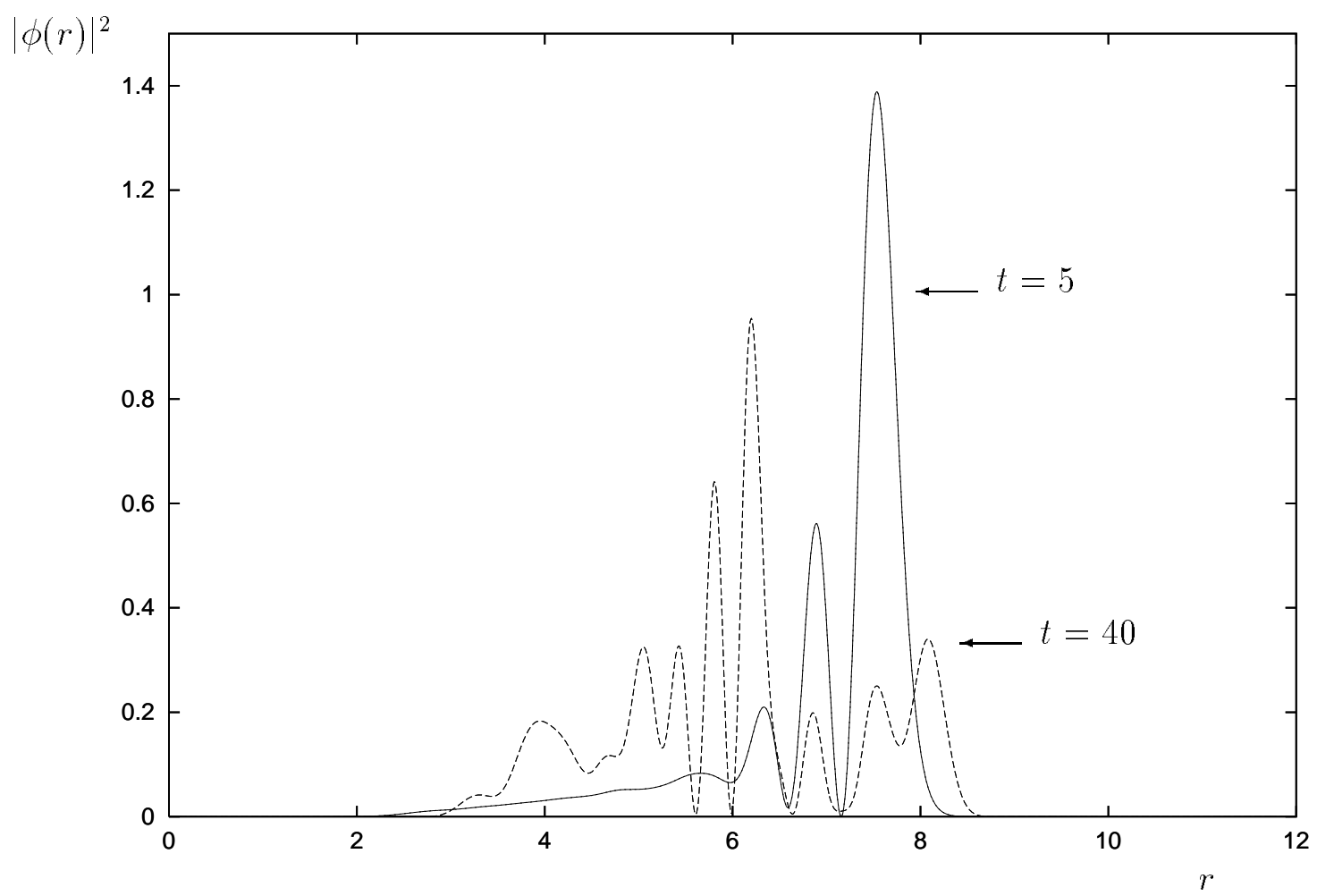

Fig. 19 